\title{
Exploratory Three Dimensional Cartographic Visualisation of Geolocated Datasets
}

\author{
A Thesis Submitted to the Victoria University of Wellington \\ in Fulfillment of the Requirements for the Degree of Master of Design Innovation
}

Alexandre Bannwarth

this thesis was completed as an online portfolio available at axbwh.thesis.surge.sh

Victoria University of Wellington

2018 


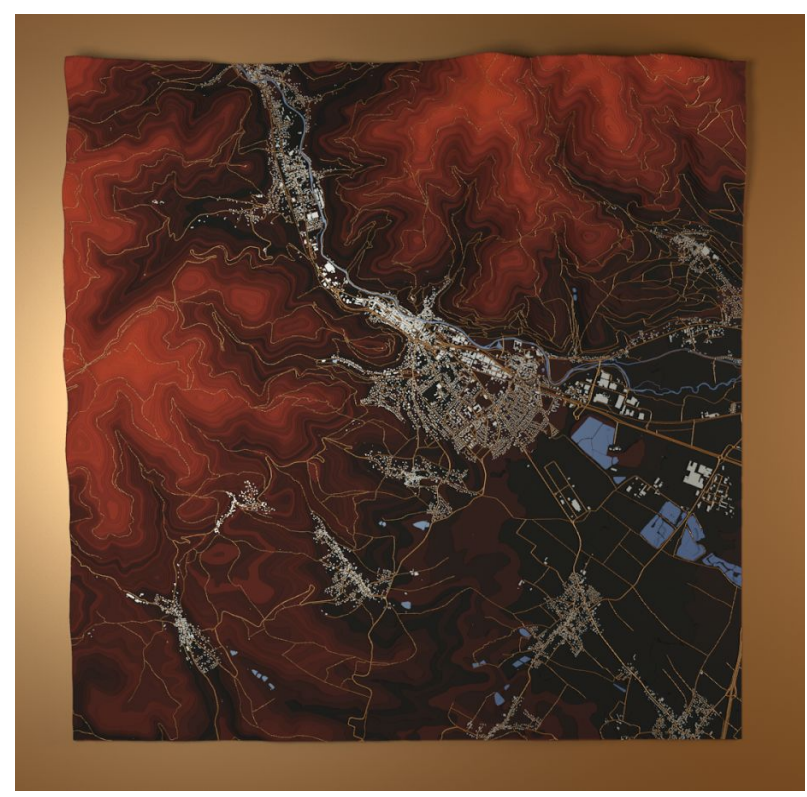

Figure 1. Thann Topographic Street Map.

\begin{abstract}
While digital technologies have vastly facilitated the generation and consumption of cartographic visualisations, the majority still conform to traditional two-dimensional map making guidelines. Consequently, design principles for three-dimensional cartographic models are still in their infancy and require further exploratory research to establish a comprehensive design framework. The free availability of high resolution global digital elevation models (GDEM), such as the ASTER GDEM (NASA LP DAAC, METI, 2011), makes it possible to develop accurate three-dimensional landscape visualisations and offer more intuitive and immersive representation of spatial information. Combined with the prevalence of geolocated content in both online data-repository and social-media platforms, there exists a wealth of material to be mined, interpreted and juxtaposed in exploratory cartographic visualisations.

This thesis aims to establish a flexible and iterative procedural workflow to acquire, interpret and visualise 3D geolocated datasets, without compromising aesthetic control. Synergic with the procedural approach required for data collection and analysis, a procedural approach is used in the design of the visual output. This workflow aims to maximise automation and allow for the interpretation of a range of different data sources. The creative output of this process explores emergent cartographic principles for optimal three-dimensional spatial visualisation and investigate data presentation techniques beyond traditional two-dimensional geo-visualisation guidelines.

Informed by exploratory literature review and research through design theories, this practice-led thesis documents the iterative prototyping leading to the creation of a set of map-based infographics showcasing effective application of cartographic and data visualisation principles in a 3D geospatial context. These design prototypes and critical report of workflow refinement hope to contribute to the development of three-dimensional geographic modelling conventions.
\end{abstract}




\section{Table of Content}

$\begin{array}{ll}\text { Introduction } & 3\end{array}$

$\begin{array}{ll}\text { Conventional Cartography } & \mathbf{5}\end{array}$

Context $\quad 5$

GIS \& Scientific Cartography $\quad 5$

Artistic Considerations in Automation \& Cartography $\quad 5$

$\begin{array}{ll}\text { Three Dimensional Cartography } & \mathbf{7}\end{array}$

Historical Precedents and their Lack of Contribution to 3D Map-Making Conventions $\quad 7$

$\begin{array}{ll}\text { Advantages of 3D Maps } & 7\end{array}$

$\begin{array}{ll}\text { Design Considerations for 3D Cartography } & 7\end{array}$

$\begin{array}{lr}\text { Data Visualisation } & 9\end{array}$

Advantages of 3D Visualisation $\quad 9$

Advantages of Infographics in the Digital Age $\quad 9$

Design Consideration for Data Visualisation $\quad 10$

Display Format for Data-Visualisation 10

Data Sources \& Social Media Mining 12

Selecting Data Sources 12

Geographic Data $\quad 12$

Social Media Data Mining 13

Methodology 14

$\begin{array}{ll}\text { Workflow } & 15\end{array}$

$\begin{array}{ll}\text { Software Selection } & 17\end{array}$

Design Process $\quad 19$

$\begin{array}{ll}\text { Inception } & 19\end{array}$

Stylised Topographic Street Map 19

New Zealand Seismic Activity Geo-Visualisation $\quad 21$

United States and Mexico Meteorite Landings Geo-Visualisation $\quad 24$

Twitter Data Mining Workflow $\quad 25$

United States Patriotic Tweets Adjusted by State Population 26

$\begin{array}{lr}\text { Conclusion } & 28\end{array}$

$\begin{array}{lr}\text { References } & 29\end{array}$ 


\section{Introduction}

With the advent of the world wide web and social media, humans are now exposed to more information than ever before in our history. Confronted with such abundance of data, individuals generally gravitate towards content based on intriguing headlines and eye-catching graphics, but will often promptly abandon even insightful documents if they are perceived to be too wordy or complex to understand. The ability of infographics and data-visualizations to condense complex information into easily shareable and palatable formats has led to an explosion in their popularity. Maps and geo-visualisation are a subset of infographics uniquely capable of presenting and elucidating cultural and natural phenomena by displaying them in relation to populations distribution, geopolitical boundaries and topographical features.

Advances in computer graphics technology have vastly facilitated the creation and consumption of a particularly immersive and intuitive type of cartographic data representation: three-dimensional geo-visualisation. This fairly niche field integrates concepts and conventions from cartography, GIS, computer graphics production and scientific visualisation (Miller \& Han, 2009) but has seen only limited contribution from the graphic and media design field. Consequently it still gravely lacks aesthetically informed conventions and precedents.

This thesis and associated creative output aspire to contribute to this lacking field through the critical development and documentation of a procedural workflow capable of processing information from varied data sources into iteratively refinable 3D geo-visualisations. This workflow also covers the acquisition of meaningful data-sets through the inspection or data-mining of various online geo-data repositories and social media sites. The research bases its validity on a thorough review of multidisciplinary literature, and a constructive, practice led approach to design research that favours tacit knowledge development and critical reflection over ethnographic assessments. 


\title{
Conventional Cartography
}

\author{
Context
}

For millennia, the art of mapmaking has been used across human civilisations to share and document geographic information. From cave painting depicting simple landscape features and star constellations to modern day digital tools like Google Maps (Google, 2005), cartography has been essential to navigating and understanding our world. This analytic function, along with the graphic consideration intrinsic to visual communication has contributed to cartography being considered a union of science, art and technology (Cartwright, Gartner, \& Lehn, 2009). This combination offers a unique potential for maps to persuade and educate (Goodchild, 2012) but also makes them prone to distort reality depending on the biases of the cartographer, the target audience or the data being displayed or omitted (Ormeling, 2014).

\section{GIS \& Scientific Cartography}

Beyond their utility as purely topographical records, maps can be overlaid with diverse data sets to visualise complex statistics and relationships. From commonplace political, cadastral (showing land ownership) or street maps, to more specialised economic or climatic ones, cartography is used within many disciplines to intuitively display geographic information. With the advent of digital technology in the 1960s, geographic information systems (GIS) have been developed to store, analyse and visualise spatial data, eventually spawning the academic discipline known as geographic information science (GIScience). Subsequently, GIS software has become an integral part of modern map-making workflows, able to output both finalised maps or layers to be refined and composited in graphic design programs.

In his paper Cartography: GIS and cartography (relationship between), Michael Goodchild (2012) details the development and convergence of GIS and cartography, stressing that although closely linked, there remains noteworthy distinction between the two fields. Of particular note is the contrast between the classical conventions of cartography and the analytical, technologically driven approach of GIS. While the first employs imagery in a way to produce easily understood, recognisable maps, the second's emphasis on precise numerical analysis often results in highly abstracted, less intuitive geo visualisations.

\section{Artistic Considerations in Automation \& Cartography}

This reliance on automated systems, along with the digital availability of geographic information technology has been criticised for eroding the artistry of traditional cartography (Cartwright, Gartner, \& Lehn, 2009). Indeed while Web-based geo-visualizations like Google Maps (Google, 2005) along with open source databases and GIS software like QGIS (Version 2.18.10; QGIS Development Team, 2017) and OpenStreetMap (OpenStreetMap Foundation, 2004) have led to a democratisation of the map-making process, authors might not necessarily be aware of cartographic conventions and constraints (Ormeling, 2014). Concurrently, many independent forays have been conducted to ostensibly re-evaluate the place of art and design in cartography. A symposium regrouping artists and cartographer responsible for such experiments is documented in Cartography and art 
(Cartwright, Gartner, \& Lehn, 2009). Of particular interest is the chapter Rejecting Illusionism: Transforming Space into Maps and Art (Fairbairn, 2009) where it is argued that while the quality of maps is primarily dependent on the faithful representation of data, there is considerable opportunity for aesthetic consideration in the interpretation of that data.

Through creative intervention throughout the map production process, it is possible to create both informative and beautiful maps, something that can be impeded by automation and technologically imposed specification. Although this phenomenon can be traced back to the printing press, in the chapter "Art and Cartographic Communication" Cartwright (2009) argues that it was offset by the high standard of completion and that such "elegant cartography" can be admired for the engraver's and cartographer's skill. By contrast, he contends that computer generated maps were promptly adopted despite subpar visuals simply due to how quickly they could be produced.

Although this trend has somewhat been averted in contemporary web-published maps through a greater design focus, Cartwright goes on to laments that the majority are still stuck in the same automation driven conventions. He argues that they fail to exploit the unconventional opportunity offered by the web medium, and that science and technology driven cartography isn't the only valid method of geo-visualisation.

It stands to reason then, that there is pertinence in a primarily aesthetic-focused, design based inquiry into innovative geo-visualisation technology. Such an opportunity exists in the nascent field of digital three-dimensional cartography. Rapid technological advances have precipitated the development and creation of 3D maps, but in the process outpacing analogous progress in the establishment of 3D specific cartographic design principles (Pegg, 2013). Indeed as conventional 2D cartographic software and conventions are unsuited for 3D geo visualisation, new guidelines will need to be devised, and will undoubtedly benefit from chiefly aesthetic consideration. 


\title{
Three Dimensional Cartography
}

\author{
Historical Precedents and their Lack of Contribution to 3D Map-Making Conventions
}

While there exist historical experiments in physical three-dimensional cartography such as tactile or braille map for the visually impaired, the specialist nature of these enquiries has made them peripheral to the development of mainstream cartography. As noted by Perkins (2002) even within such a highly specialised field, there is a lack of research into appropriate design guidelines for thematic tactile mapping. The constraints of conventional three-dimensional manufacturing methods like etching, embossing and vacuum-forming have also made general adoption of physical 3D (three dimensional) maps unviable.

Another classic application of 3D maps can be found in ski resort trail maps, where skiing and hiking trails are depicted over mountain terrain in artistic 3D perspective illustrations, often doubling as eye-catching advertisement in promotional material. While these panoramic maps do not suffer from the manufacturing challenges of tactile maps, their method of production remains more time and cost intensive than standard $2 \mathrm{~d}$ maps (Schobesberger \& Patterson, 2007). Indeed such birds-eye view representations were traditionally painted or illustrated by highly skilled cartographers using topographic maps and aerial photography as reference (Pegg, 2013). Even with 3D software making this task significantly faster, such panoramas still require specialised software unfamiliar to most modern cartographers. The creative challenges and artistic opportunities offered by three-dimensional perspective, along with the advertising considerations needed to produce such ski resort maps have also limited their prevalence. This niche nature has lead to a lack of contribution to common 3D mapping principles (Pegg, 2013).

\section{Advantages of 3D Maps}

With the arrival of free high resolution global digital elevation models (GDEM), such as the ASTER GDEM (NASA LP DAAC, METI, 2011) and processing power permitting 3D perspective view in web-based geo-visualisation tools like Google Maps (Google, 2005), there is now unprecedented interest in 3D maps (Schobesberger \& Patterson, 2007). Beyond the novelty and technology factor, this growing popularity can be attributed to the intuitive depiction of landscapes and expressiveness offered by 3D maps (Häberling, Bär, \& Hurni, 2008). As explained in Evaluating the effectiveness of $2 \mathrm{D}$ vs. 3D trailhead maps (Schobesberger \& Patterson, 2007), the way traditional maps depict terrain elevation through contour lines, shaded relief or height points can prove unintuitive and confusing to inexperienced map readers. Visualising and associating 2D cartographic representations with real world landscape features can be difficult for large segments of the population, in three dimensions, however, panoramas closely resemble reality and offer a familiar understanding of elevation and topographic features. With overlaid trail info, the realism of 3D maps makes it easier to identify one's location, particularly in mountainous terrain. Contrarily, however, Schobesberger and Patterson (2007) found recollection of location names was more difficult with 3D maps, Pegg (2013) suggest this might be due to subpar or inappropriate symbology. 


\section{Design Considerations for 3D Cartography}

Due to the lack of adequate 3D cartographic design conventions, how to best take advantage of the immersiveness of 3D maps remains an area of contention. In Proposed cartographic design principles for 3D maps: a contribution to an extended cartographic theory, Häberling et al. (2008)delineates aspects of 3D cartography in need of specific design principles. To produce intelligible, interpretable and harmonious map images, specific directives need to be adhered to throughout the cartographic design process. These directives will vary depending on conceptual, technical and product requirements. The intention and circumstance of utilisation along with the user's familiarity with cartographic visualisation will define conceptual aspects, while digital input data, medium of delivery and software choice will dictate technical ones. Finally, product requirements will depend on the thematic content, usability and visual appeal of the 3D map.

Keeping in mind these considerations, Häberling et al. (2008) define the 3D cartographic process as an iterative workflow comprising three phases: modelling, symbolisation and visualisation. Modelling comprises the transformation of DEM and other geodata into 3D models, as well as the modelling of map objects and labels. Symbolisation covers the design of graphic appearance, textures and animation, while visualisation encompasses perspective and camera settings, lighting, shading and atmospheric effects. These stages can essentially be equated to the modelling, texturing and rendering phases in a conventional CG workflow.

Based on this workflow and referencing Pegg (2013)'s Design issues with 3D maps and the need for 3D cartographic design principles we can identify pivotal design dilemmas. A fundamental decision lies in the degree of photo realism or abstraction to display. In the modelling phase, the level of realism is determined by the application of map projection, while essential in conventional 2D maps to transform latitude and longitude values from an ellipsoid to a plane, 3D makes exact topographic reproduction possible instead. Depending on the scale and application of the geo-visualisation however, conventional projection might be preferred to distortionless globe displays.

Symbols and labels can also display varying levels of photorealism, buildings and landmarks can be modelled to closely match reality or instead use abstracted generalised markers. While the first option might be preferred for a visually striking, highly detailed small scale urban geo-visualisation (Mao, 2011), re-using easily recognisable markers might prove more cost efficient and more legible in larger maps. Likewise, in the texturing phase, one should consider whether to overlay photographic images to produce realistic landscapes or instead favour stylised thematically coloured textures to graphically display information over the 3D surface (Häberling et al. 2008).

To ensure legibility these choices need to be considered in unison with the typesetting of text labels and colour design, ensuring appropriate contrast and harmony in the design of the colour scheme. Finally, the choice of viewing angle and illumination are identified as critical in the legibility of 3D map products, as varying camera and lighting angles may distort or obstruct perception of distance, topographical features, labels and symbols. As all these factors need to work in harmony to produce effective 3D maps, ideal software and workflow should allow for rapid iterative prototyping without restrictive pre-determined automation. This can be done by adapting commercial 3D visualisation software for architecture or computer graphics (Häberling et al. 2008). 


\title{
Data Visualisation
}

\author{
Advantages of 3D Visualisation
}

Although most literature on 3D cartography focuses on street or trail maps, the benefits of three-dimensional visualisation aren't limited to purely navigational aids. 3D Visualization offers improved insight into complex data-sets as information can be displayed and interpreted more effectively (Helbig et al. 2014; Pegg, 2013; Bandrova, 2005). 2D limits the number and ways in which variables can be visualised and combined, on an $x-y$ plane data representation is restricted to two-dimensional shapes that once overlaid to compare multiple datasets can become cluttered and unintuitive. The use of colour coding, symbols and chart legends mitigates this effect, but at the expense of superfluous eye movement. Constantly looking back and forth between color key and the visualisation make them more laborious to decipher and should be minimised (Krum, 2013) The addition of a third $z$ dimension offers the possibility to intuitively display variables as both spatial location or height component of solids, potentially resulting in distinct 3D elements. The third dimension can also be used to hierarchically organise data-sets into layers, or accurately and intuitively contextualise thematic information, for example, meteorological data being visualised as volumetric elements above a 3D landscape. In this way, 3D makes it possible to superimpose data from various sources and visually evaluate their relationships, highlighting correlations and phenomena (Helbig et al. 2014). Moreover, errors and discrepancy in the data are easier to identify in 3D, for example, artifacts in the ASTER GDEM (NASA LP DAAC, METI, 2011) can be hard to catch as they appear as overbright pixels in the grayscale heightmap, but once turned into a 3D mesh they unmistakably stand out as absurdly distorted geometry.

\section{Advantages of Infographics in the Digital Age}

Beyond considerations specific to three-dimensional cartography, 3D geo-visualisations should also be cognizant of best practice for data-visualisations and the wider field of infographics design. While the terms infographics and data-visualisation are often used interchangeably, Krum (2013) points out that in recent years the terms have evolved more explicit meanings to infographic designers. He defines data-visualisation as an umbrella term for any visual representation of numerical values (graphs, maps, charts, etc.) while the term infographic has come to signify a composite graphic design making use of data-visualisations, text, images and illustrations to create a narrative. This distinction reflects the novel ways in which we consume data-visualisation, just as web technologies have revolutionised maps, so has social media fundamentally changed how infographics are shared and designed.

Infographics have long been utilised to quickly and efficiently communicate information. Taking advantage of human propensity for pattern recognition and picture retention, data-visualisations condense and organise large data sets into concise, memorable graphics, highlighting patterns and trends (Miller \& Han, 2009; Krum, 2013). As the cognitive phenomenon known as Picture Superiority Effect dictates that images and graphics are more likely to be remembered than words (Nelson, Reed \& Walling,1976), it is easy to understand the popularity of infographics. Until recently, however, the majority were designed for print media, limited to newspapers, magazines, textbooks, etc. and did not garner the interest that web-publishing has allowed for. Being presented with more information than ever before(Hilbert, 2012), web-users swiftly determine what is worth their time and frequently 
switch between topics of interest (Weinreich, Obendorf, Herder, \& Mayer, 2008), making it crucial for critical information to be communicated succinctly and enticingly. Moreover, the way information is increasingly disseminated through social media favours self-contained display format that can easily be shared and consumed on various platforms. As designers have had to adapt their messaging to these new browsing habits, the popularity and public awareness of infographics and data-visualisations has seen rapid growth (Smith, 2013; Krum, 2013).

\section{Design Consideration for Data Visualisation}

Following this explosion of online infographics, an abundance of designs of varying quality now permeates the web. Krum (2013) identifies a number of factors necessary in the design of successful web-infographics. Distinctive and aesthetically appealing graphic design is paramount for any infographics to capture and hold the attention of prospective consumers. The infographics might only be onscreen for a short time as users scroll down news feeds or quickly switch between tabs (Weinreich et al. 2008), or be displayed as a thumbnail, making eye-catching visuals critical in enticing users to find out more. Infographics incorporating unique and innovative visualisations are also more memorable and more likely to be shared through social media, further validating the pertinence of experimentation with unconventional 3D visualisations.

Krum (2013) also identifies the brevity and legibility as important factors in the success of infographics. As the majority of readers only skim through web documents for a few seconds (Weinreich et al. 2008), he suggests that the central message should ideally be able to be understood in 5 seconds or less. This means essential information should be prominently featured in the design, while unimportant or irrelevant information should be compositionally minimised or altogether omitted. Superfluous text detracts from the appeal of infographics, as users will perceive excessive text to be compensating for sub-par visual communication. Infographics must thus rely on data-visualisations to reduce the time needed to understand data, provide context through visual comparisons and make the design more memorable. Good data-visualisation need to be clear and easy to understand for a lay person, striking a balance between precision, simplicity and relevancy. If a data-visualisation appears too complicated to understand, most readers will abandon it regardless of the veracity of the data.

\section{Display Format for Data-Visualisation}

Advances in multimedia and web-technologies continuously increase the diversity of formats infographics and data visualisation can take, from static images to adaptive web-pages and fully interactive visualisation tools. Varying display formats offer advantages and disadvantages in the way data is consumed and shared (Bellato, 2013). Full blown interactive visualisation tools usually provide the most compelling and in depth examination of data at the cost of lengthy development time, device restrictions, require prolonged user engagement and cannot be directly embedded on social media platforms (Krum, 2013). Similarly, animated web page infographics utilise html, css and javascript to enliven the graphics with animated and clickable elements but also suffer from inferior shareability. Video infographics, although predating the internet age, are gaining unprecedented popularity due to how easily they can be shared and embedded on video sharing sites and social media (Krum, 2013). They smoothly communicate information in clear narrative sequence, presumably complementing the visual elements with music, voiceover and animation, further facilitating the decipherment of the information presented (Bellato, 2013). Finally, static infographics 
are the simplest to produce and distribute as image or PDF files, easily embeddable and shareable on websites and social platforms while also readily adaptable for print media (Krum, 2013). The relative swiftness of the production of these static infographics allows for faster prototyping and revisions in an iterative workflow. Although efficiently taking advantage of web pages' infinite scrolling, the tall format adopted by many static infographics is impractical for presentation slides or physical distribution.

The successful proliferation and popularity of these static infographics have led to the emergence of a sub industry focused on the design and online sale of large format infographics posters. This business model allowing for greater creative freedom in the choice of topic and aesthetic style, enabling designers to create visualisations and infographics based on personal design sensibilities (Krum, 2013). 


\title{
Data Sources \& Social Media Mining
}

\author{
Selecting Data Sources
}

Of utmost importance in the success and integrity of infographics is the credibility and accuracy of the information visualised (Krum, 2013). Data sources must be carefully curated to ensure the provenance, relevance and newness of the data. Establishing that the data originated from reliable sources, and was generated through legitimate methods is mandatory when collecting data. For readers to trust the infographics and share it on social media, they must be confident in the reliability of the information; the output design must thus reflect that the designer has done their due diligence. Krum (2013) urges designer to locate and explicitly list and date the source of the data in the design itself or as a reference in the footer. Indeed as most readers won't go to the trouble of verifying the sources themselves, it is important for self-contained infographics to project a semblance of legitimacy.

\section{Geographic Data}

The variety, scope and accuracy of digital geographic datasets have seen fast and continuous growth over the past few years (Miller \& Han, 2009). Private companies and government agencies utilise advanced spatial sensing systems to collect large, highly detailed digital geodata on various subjects, such as topography, infrastructure, land-use, socioeconomic conditions, seismic activity, etc. The propagation of global positioning systems (GPS) in phones, in-vehicle navigation systems, and other smart-devices also enables unprecedented collection of geolocated data, either through proprietary software or data-mining geotagged social media activity. These data-sets are often freely disseminated through an ever-growing number of online geographic data repositories, facilitating the acquisition and juxtaposition of data to be visualised.

The data on these repositories come in a variety of context-appropriate multimedia formats that can be retrieved through either site-specific Application Programming Interfaces (APIs), data-mining methods, web-scraping or manual download. Miller \& Han (2009) stipulate that to uncover meaningful patterns in geographic data sets, a human-centered, nonlinear iterative process must be employed. This process typically consists of several stages that may be consciously omitted or revisited depending on the data being processed, the desired knowledge and intermediate results gleaned throughout the data-mine and visualisation process. It can be divided into the following pivotal steps: Data selection consists of the election of a specific subset of variables in the dataset that are of particular relevance to the intended geo-visualization. Data preprocessing aims to clean up any noise, artefact or redundant information in the data and reformat it if necessary. Data enrichment involves the addition or juxtaposition of supplementary data fields from complementary data sources. The Data-mining stage concerns itself with exposing concealed patterns in the data. Finally, the interpretation and reporting stage involves critical assessment and communication of the uncovered information.

While automation is essential to individual steps in this process, Miller \& Han (2009) stress the importance of persistent human problem solving throughout the workflow as machine-based approaches have not yet surpassed human cognition for many complex tasks. By exploiting human 
visual acuity and pattern recognition, visualisations are the ideal way to effectively integrate high-level human intelligence into this data discovery process. Consequently, geo-visualisation should not be considered as mere visual outcomes or display technology, but as fundamental tools in the pattern discovery and interpretation stages of the process. Effective geo-visualisation techniques facilitate the uncovering of geographic concepts and processes and help formulate novel geographic queries.

\section{Social Media Data Mining}

As mobile devices now account for over $80 \%$ of social media activity (ComScore, 2017), and with the ubiquity of GPS in mobile devices, many social media platforms now encode geolocation into the metadata of user-created content. Such geotagged content now enable us to map social media habits and highlight potential correlation between geographic location, trending topics and other geolocated datasets. The broad appeal and increasing popularity with individuals from every walk of life have made social media a unique source of user-generated data (Russell, 2013). The shear rate at which data is generated makes social media mining uniquely equipped for observing and understanding large scale human behavioural patterns. Effectively harnessing the enormous potential of social media data requires new tools and technologies to be developed to represent, analyse and extract actionable patterns from these unconventional user-generated data types. (Zafarani, Abbasi \& Liu, 2014)

Mining social media data comes with some distinct challenges in contrast to conventional datasets. Despite the overall volume of data, often only meager or incomplete information can be obtained from specific individuals or posts, either due to a lack of activity or technical limitations. Indeed many APIs for social media platforms only enable a limited amount of data to be mined daily, while privacy settings may further restrict data available to extraneous queries. For example, the twitter API only searches tweets no older than 6-9 days. It is thus important to be aware that samples may not be representative of the full data and underlying patterns. This phenomenon can be further exacerbated by the noise and unstructured nature of user generated data creating unique challenges in data selection and data preprocessing. Indiscriminate noise removal may further skew the integrity of the data, or unintentionally eliminate relevant information as noise must be arbitrarily defined when evaluating unorganised, extemporaneous qualitative data. (Zafarani, Abbasi \& Liu, 2014) 


\section{Methodology}

Currently, no single design conventions or methodology definitively account for the synthesis of data-mining, cartography and three-dimensional visualisation undertaken in this thesis. More-so due to the comparative immaturity and consequent lack of precedents for 3D Geo-visualisation. While conventions and design guidelines can be extrapolated from traditional 2D cartography and generalised from data-visualisation and infographic design, a review of precedent literature only offers limited insight into actionable steps required in a successful 3D Geo-visualisation process. Nonetheless, a literature review was undertaken to establish existing theory relevant to this project, and contextualise the study within this body of knowledge (Creswell, 2013). Of particular interest were texts delineating concrete workflows and technologies that could be combined or adapted into a flexible, iterative process, able to incorporate and visualise in 3D diverse types of geodata.

As previously established, the niche this research falls into, namely 3D data geo-visualisation, has only emerged relatively recently as an amalgamation of elements from various fields. It did so largely due to the introduction of new technologies and remains relatively underdeveloped. Consequently, the interest of this study relies heavily on the inventive combination of pre-existing ideas and how to take advantage of these emergent technologies, factors identified by Norman and Verganti (2014) as fundamental to radical innovation. In contrast to human centred design processes that involve extensive ethnographic research and usability testing throughout the process (Cooley, 2000), radical innovation arises from mostly independent exploration and experimentation with novel technologies to produce seminal products (Norman \& Verganti, 2014). While user centered design is essential to the prolonged evolution, incremental refinement and lasting success of these products it has been argued to be unnecessary and even potentially limiting to the initial ideation and development (Norman \& Verganti, 2014; Norman, 2005). Although successful attempts at radical innovation are rare, they generally emerge from the cumulative knowledge and theories generated through failed attempts (Norman \& Verganti, 2014).

This reflects the increasing recognition and endorsement of design as a knowledge generating activity (Durrant, Vines, Wallace, \& Yee, 2015). Indeed, the concept of research through design envisions design practice as a generative and iterative process, that can formulate theories and participate in the construction of possible futures through tangible, relatable design artefacts (Durrant, Vines, Wallace, \& Yee, 2015; Gedenryd, 1998). Employing such a practice-based approach to design research allows practitioners to codify the tacit knowledge (or know-how), acquired throughout the construction of prototypes, products or models (Koskinen, Zimmerman, Binder, Redstrom \& Wensveen, 2011).

While there is still much debate surrounding research through design and the rigor of practice-led knowledge (Durrant, Vines, Wallace, \& Yee, 2015; Biggs \& Buchler, 2007) through an aggregation of concepts from practice-based research, action research and grounded theory, Godin and Zahedi (2014) summarise criteria instrumental to the validation of such knowledge. They highlight the importance of recoverability as a substitute for reproducibility, since due to the creative nature of design practice, even given an identical problem and framing, designers will seldom produce identical results (Zimmerman, Forlizzi \& Evenson, 2007). Instead, recoverability implies that the design process is documented in such a way as to be understood and critically scrutinised (McNiff, 2013). Concretely Godin and Zahedi suggest that the design process should be recorded clearly, 
succinctly and in chronological order (Pedgley, 2007) while also demonstrating theoretical sensitivity which is defined as the researcher's capacity to use personal and professional experiences, in addition to the literature, to innovatively explore and develop theories from the research situation (Strauss \& Corbin, 1990). Finally, they identify the adequacy and good functioning of the produced artefact as validation of the generated knowledge (Findeli, 2003).

Given these considerations, this thesis positions itself as practice-led, constructive design research centred around the development and critical documentation of an emergent procedural workflow for 3D geo-visualisation. For the workflow to handle diverse and large data sets, be repeatable and allow for rapid iteration, it aims to maximise procedural automation but without compromising artistic control. It is refined through an iterative design process, progressively creating and improving compartmentalised procedures to transform a range of geo-data into 3D maps. As a range of visualisations of varying scale and theme are iteratively produced and polished, the workflow and procedures are tested and evaluated. Since geodata come in diverse formats that need to be processed and displayed differently, the workflow must allow for the flexible reuse and adaptation of these compartmentalised procedures.

\section{Workflow}

Based on Häberling et al. (2008)'s cartographic process and Miller \& Han (2009)'s data mining stages, the workflow is divided into the following phases.

Data selection: Identifying and acquiring interesting data-sets from online geo-data repositories and social-media platforms. Done through the use of site-specific API, web-scraping or manual download depending on the type of data and web-access.

Data preprocessing: Initial clean up of noise and artefacts in the data, and conversion into software-friendly file formats. Done through scripting or format-dependent software tools. At this stage, data-sets can potentially be enriched by appending or combining complementary data.

Modelling: Transformation of geo-data into three dimensional models, and the creation of additional objects and labels. Undertaken within a 3D procedural generation software, this phase is key to discover and expose patterns in novel and unconventional ways.

Stylisation: Establishing the graphic appearance, materiality, textures and colour scheme of the geo-visualisation inside the 3D software. Can rely on both procedural texturing and the application of raster geo-images.

Visualisation: Determining light and camera setup, viewing angle and render format. External renderer plugin can be utilised for more comprehensive settings and superior quality.

Post-Processing \& Infographic Design: Optional refinement and integration of the geo-visualisation into a broader infographic format. Executed in 2D graphic design software.

It must be noted that this process is nonlinear and highly iterative as multiple phases might be executed concurrently. For example, the viewing angle will affect the composition of 3D elements, just as lighting setup will influence the materiality and colour scheme. Noise in the data might also 
only become apparent once translated into 3D meshes and need to be removed through data preprocessing. 


\section{Software Selection}

Considering the idiosyncrasies of the outlined workflow, its proficiency will depend on the selection of versatile and robust software tools, most important of which is the choice of 3D application. As suggested by Häberling et al. (2008) commercial programs from architecture, manufacturing or computer graphics can be adapted for 3D cartography. Since the researcher's expertise lies in graphic design and 3D modelling, the later option was obviously preferred, and a number of industry-standard 3D applications were considered following a few key criteria. Ramachandran and Varoquaux (2011) note that visualisation tools should integrate both graphical interface and full-featured scripting to allow for the visual examination, tweak and transformation of complex data-sets into suitable visualisations. Being crucial to the workflow then, procedural capabilities of potential software were reviewed. While most allow for scripting via python or proprietary language such as Maya Embedded Language (MEL) in Autodesk Maya (Version 2017, 2017), the packages were predominantly conceived and structured for more linear workflows.

Side Effects Software's Houdini (Version 16.0.557; 2017) stood out as every aspect of the program is designed for proceduralism. Modelling, texturing, shading and rendering are all done through a node based visual programming interface, where operators can be connected into complex networks to construct digital assets. Data manipulation is fundamental to this procedural paradigm, whether it is imported or generated, data can easily be accessed, visualised and modified for any node along the network. Packaged operators can be combined into user-defined meta-operators called digital assets and instanced into larger networks, allowing for the interactive development of sophisticated tools. Custom operators can also be coded in VEX, Houdini (SideFX, 2017)'s highly optimised scripting language. Nodes can be modified or rearranged at any point, allowing for the networks to be iteratively refined, or reused. During longer projects, libraries of procedures can be created in this way and used to automate tasks. Houdini (SideFX, 2017)'s combination of visual and conventional programming makes procedural generation more accessible to 3D artists, and the Graphical User Interface (GUI) offers familiar and intuitive graphic control. Moreover, it's support and integration of industry standard 3rd party rendering software such as Solid Angle's Arnold (Version 2.0.1; 2017) enables extensive procedural control over the final visual outcome.

The artistic and algorithmic flexibility afforded by Houdini (SideFX, 2017) make it ideal for this project, as it can import and process diverse data formats. It is not however designed to handle geospatial file encoding, thus requiring specialised software to pre-process and convert data into a supported format. The free and open-source geographic information system application QGIS (2017) adequately fills this role as it supports the editing, composition and exporting of vector and raster geodata. It is especially useful in stitching together numerous raster images, filling voids in the data, and converting files from disparate sources to a standard cartographic projection.

As previously noted, the tools necessary to mining data-repositories and social media will vary based on available APIs, site policy and data-type. However, Python programming language is recurrently suggested in data-mining and visualisation literature due to its intuitive syntax, optimisation and extensive range of packages facilitating API access, web-scraping and data manipulation (Russell, 2013 ; Ramachandran \& Varoquaux, 2011). Python scripting is also instrumental in pre-processing and formatting large data sets into spreadsheet format ready to be passed to a table-import node in Houdini (SideFX, 2017). 
Post processing and auxiliary 2D graphic design can be undertaken in prominent design application such as Adobe Illustrator and Photoshop, as they offer better control and agility in designing context specific compositions. 


\title{
Design Process
}

\author{
Inception
}

To better contextualise the following process report, it must be noted that this project stems from prior research and experimentation with procedural visualisation. While this precursory project focused on visualising human biometric data rather than geospatial information and was conceived as an abstract real time VR experience, in terms of design practice and tacit knowledge it is a direct antecedent to this thesis research.

Initial experimentations were conducted within Unreal Engine (Version 4.16, 2017)'s node based visual coding environment, whose procedural implementation shares many similarities to that of Houdini (SideFX, 2017). The aim of the prototype was to create complex three-dimensional visuals responding in real time to a biofeedback datastream. Although some interesting abstract effects were achieved by instancing and procedurally animating 3D solids, it quickly became apparent that the constraints imposed by real time rendering were too limiting.

This realisation led to a search for new software that could be utilised to procedurally model complex real time assets, effectively minimising runtime computation. Thus, Houdini (SideFX, 2017) was selected, but the depth, flexibility and incredible potential of the software were found to easily warrant its investigation as a primary design environment. However, the shift to a non-real time engine meant only pre-recorded biometric data could be visualised, significantly reducing their appeal.

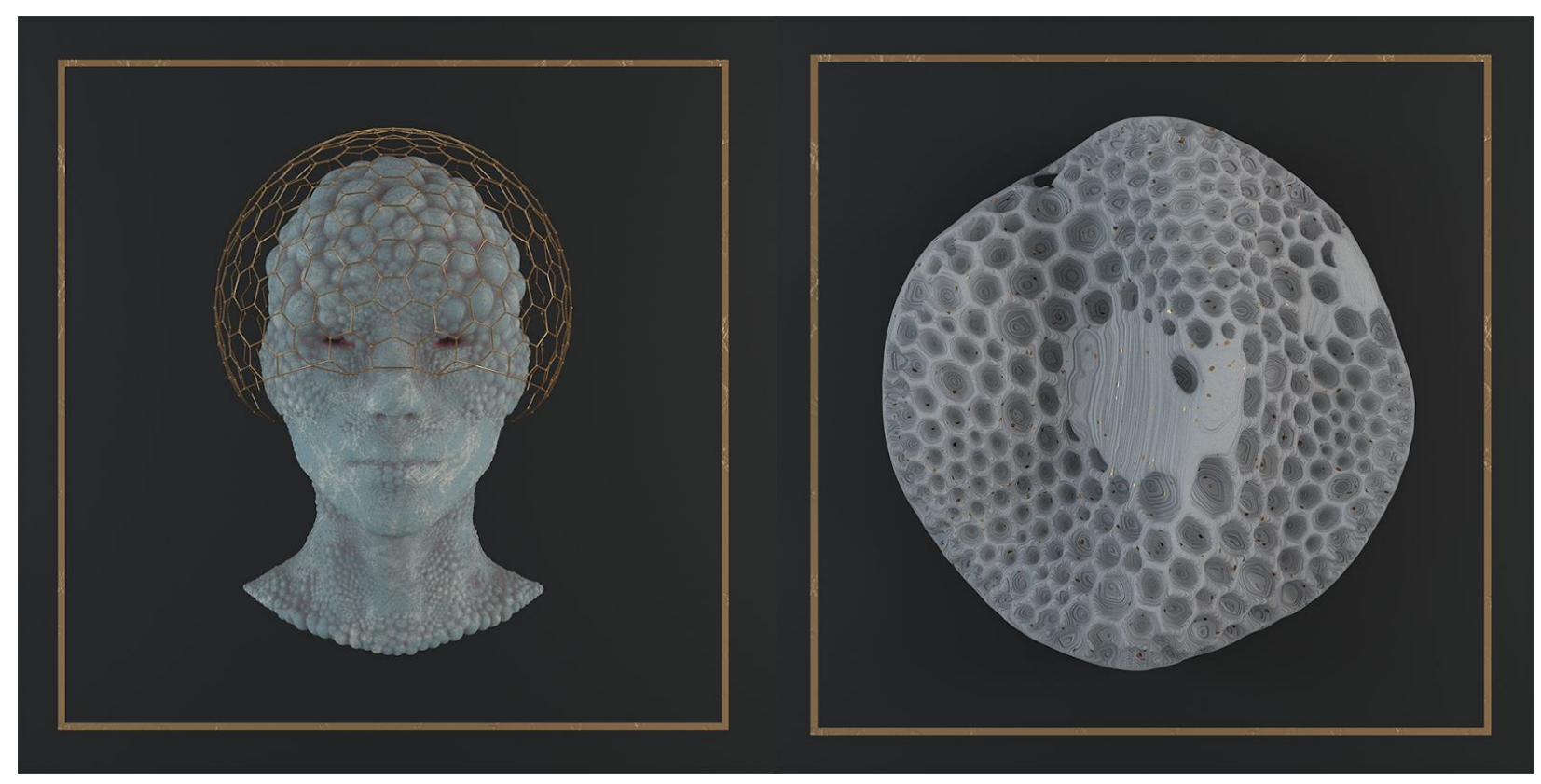

Figure 2. Houdini Generated Artefacts. 
Practical investigation and skill development continued, and through manipulating data and variables in 3D space to procedurally construct highly complex and aesthetically intriguing artefacts, new possibilities became apparent.

While the data for these early experiments was either imported as geometry or generated through turbulence maps and procedural operators, it quickly became evident that the same methods could be used to manipulate more meaningful data. Experimenting with the powerful Arnold (Solid Angle, 2017) render engine, the aesthetic quality that could be achieved further convinced the researcher to focus on producing non-real time but highly finessed elements.

These discoveries, combined with an interest in the visual qualities of cartography and panoramic visualisations as well as a fascination with the cultural meaning we draw from maps convinced the designer to conduct preliminary research into 3D geo-visualisation. A gap in knowledge and corresponding opportunity were promptly identified, leading to a shift in context and methodology. The core concept of exploratory data-visualisation remained constant however, as did the coding and procedural development expertise.

\section{Stylised Topographic Street Map}

For an initial foray into the implementation of a 3D geo-visualisation workflow, a large scale, stylised panoramic map seemed ideal. Focusing on the fundamental elements of 3D cartography, this map took inspiration from trail maps and physical dioramas to creatively visualise common topographic and urban elements.

To do this effectively a region that showed a good mixture of mountainous terrain, streets, paths, buildings and bodies of water was selected. The town of Thann, birthplace of the researcher, sits at the Entrance of a valley in north eastern France, flanked by rolling hills and bisected by the Thur river. From a compositional standpoint, the contrast between open-farmland, hilly terrain, urban centre and the smaller villages dotted around the landscape showed a lot of promise.

\section{Data selection}

Acquiring accurate elevation data was a primary concern as the large scale would highlight low res meshes. Many Digital Elevation Models are available on the web, some of which can be browsed through the United States Geological Survey's EarthExplorer (USGS, 2017) web application along with a wealth of other freely available cartographic data. The ASTER GDEM (NASA LP DAAC, METI, 2011)'s worldwide coverage, and relatively good resolution made it the most appropriate for this location. The elevation data comes in a mosaic of grayscale heightmap GeoTIFF images 3601px squared, spanning one longitudinal degree by one latitudinal degree. The elevation is encoded into the luma channel of the images, lighter values corresponding to a higher altitude. As the town spans the junction of only two of these fragments, they were manually downloaded through the EarthExplorer (USGS, 2017) database. The open source access, satisfactory accuracy and QGIS (2017) integration of OpenStreetMap (OpenStreetMap Foundation, 2004) made it an ideal source of vector data for roads, buildings and waterbodies. Quality volunteer created vector data for the entire world can be downloaded, processed and exported directly from the QGIS (2017) interface. 


\section{Data Preprocessing}

As disparate data-sources come in a range of different map projections, a common one had to be decided upon. The web-standard Pseudo-Mercator projection was elected, as it is most prevalent in daily living and its distortion of countries and geographical features is familiar to the majority of the population.

Before being taken into Houdini (SideFX, 2017) the ASTER DEM (NASA LP DAAC, METI, 2011) images had to be stitched together, cropped and projected, this was easily done thanks to QGIS (2017) specialised tools and extensive map-projection library. OpenStreetMap (OpenStreetMap Foundation, 2004) vectors were curated and whittled down to elements that would contribute to a minimalist cartographic aesthetic. They were then cropped and exported to Illustrator 8 format (AI) as only EPS encoded files are natively supported by Houdini (SideFX, 2017).

\section{Modelling}

In order to transform imported heightmaps into 3D geometry, a straightforward self-contained node network was built to create a polygonal grid of adjustable resolution and extrude it depending on the luma of the image input. Despite the ASTER GDEM(NASA LP DAAC, METI, 2011) being the highest available resolution for the region, when working at such large scale, the pixel size of the GDEM limited the realism of the mesh created. This technical limitation motivated the development of a wood-cut map inspired aesthetic, also reminiscent of contour lines from 2D map making. To do so, a custom network was built to take any 3D mesh and procedurally slice it based on either segment height or total number of slices.

Roads and building were then imported as a set of vector files and procedurally projected onto the irregular surface and extruded, further contributing to the diorama aesthetic.

Each of these steps was designed wholly or in part as reusable meta-operators. While a lengthier process than standard linear modelling workflow, the upfront time investment pays dividend as a library of automation is built up.

\section{Stylisation}

Complementing the stylistic choices made during the modelling process, the colour scheme and materials were picked to be thematically relevant to the town being visualised. Indeed the colours were based on the coat of arms of the city, consisting of a golden fir tree on a field of blue, split with a white stripe on red. Thus the golden roads, blue rivers and lakes, and white buildings on a red landscape. The colour and brightness of the landscape was procedurally influenced based on height component and slightly randomised to give a more organic stratified soil aesthetic. 


\section{Visualisation}

A few different viewing angles were experimented with, the most interesting of which was a moderately high angle shot looking over the farmlands and down the entrance of the valley, making for quite a dramatic panorama. Despite bird's eye view arguably defeating the purpose of three-dimensionality, such renders proved very visually compelling nonetheless. Indeed with proper camera settings, some depth perception is maintained, and a subdued light setup produces soft shadows and burnished reflections, making for a very aesthetically pleasing map.

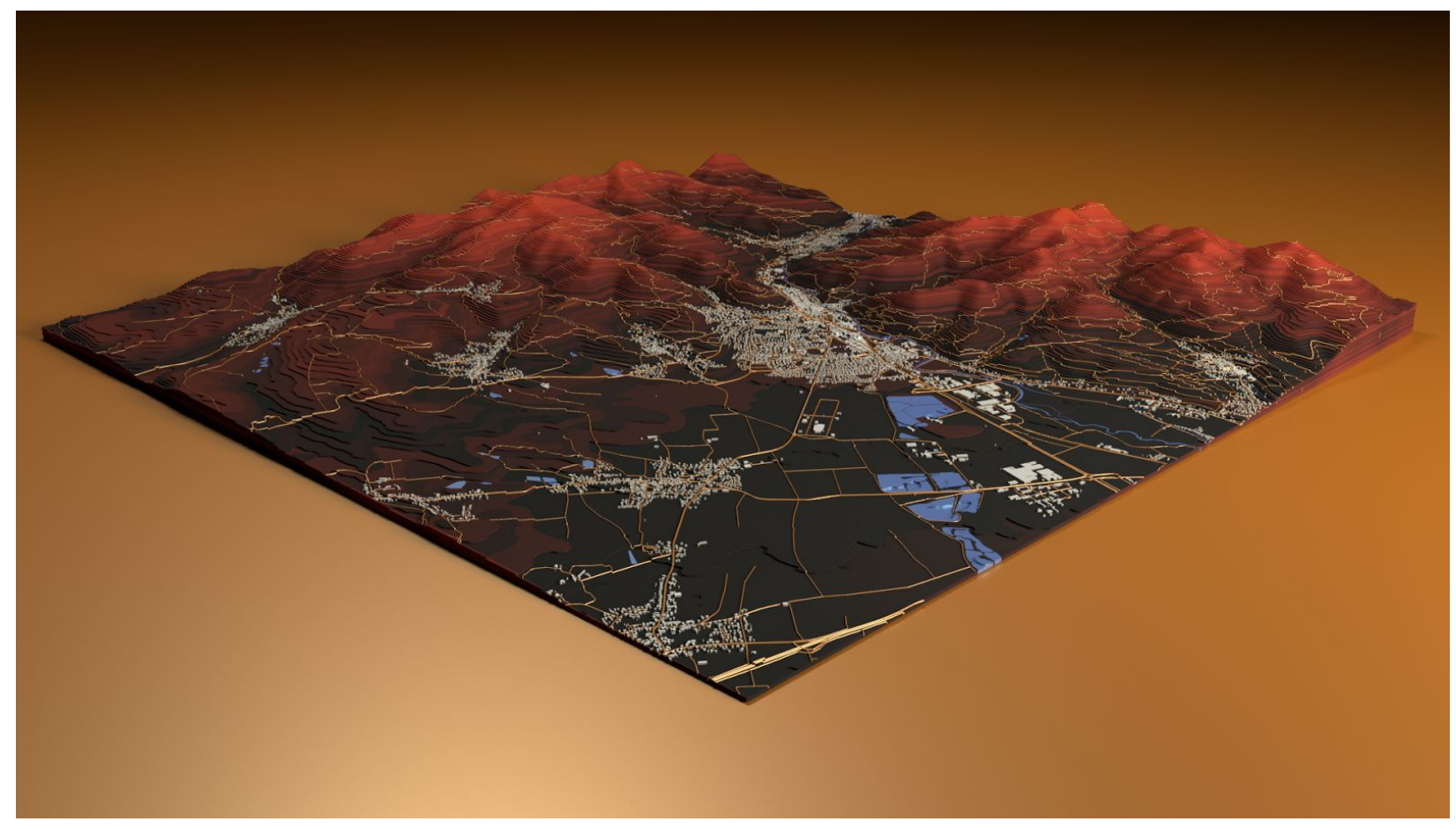

Figure 3. Thann Topographic Street Map Panorama. 
The visual quality of the map renders was persuasive enough to warrant the choice of a series of large format cartographic posters and digital wallpapers as creative outputs.

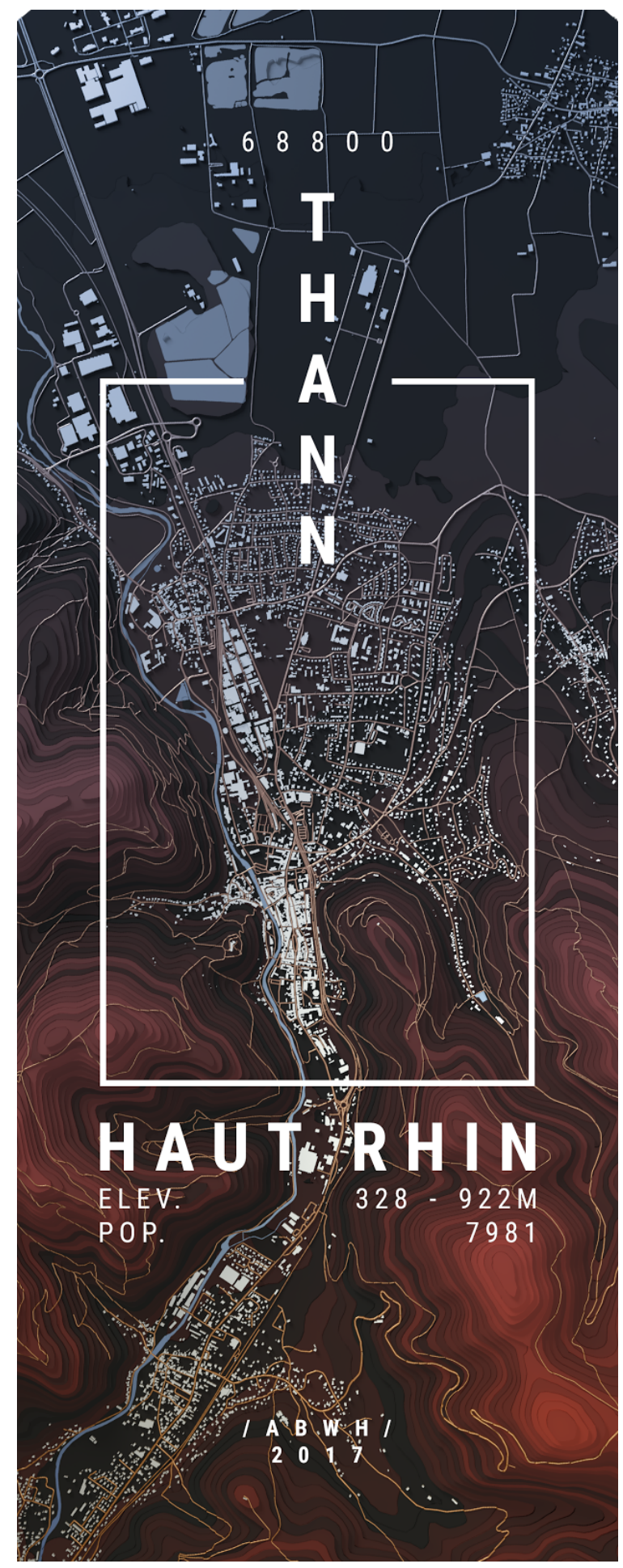

Figure 4. Thann Topographic Street Map Poster. 


\title{
New Zealand Seismic Activity Geo-Visualisation
}

\author{
Data Selection
}

Having established a functional workflow for acquiring, and processing core data-types, potential auxiliary geotagged data-sets were investigated. The GeoNet Earthquake Catalogue (GNS Science, Earthquake Commission, 2017) was identified as having a lot of potential for 3D visualisation as some variables could be visualised along the $y$-axis. While there exists a Geonet API, a simple search query can return earthquake data as a CSV table, Houdini (SideFX, 2017)'s format of choice for data import.

ASTER GDEM (NASA LP DAAC, METI, 2011) data for New Zealand was again acquired through the USGS EarthExplorer (USGS, 2017). The 63 individual height maps were downloaded using their proprietary batch downloading software. This process was made very tedious by the EarthExplorer (USGS, 2017) user interface's limited support for batch selection and the tremendously unoptimised downloader, that unjustifiably utilised up to $95 \%$ of the CPU, effectively monopolising a machine for the lengthy download. Clearly, an alternate solution would have to be devised for future batch downloads.

As the elevation data does not make coastlines clearly visible, vector data for country boundaries was obtained from the Natural Earth (NACIS, 2017) website, an online repository offering free access to vector and raster map data at various scales.

\section{Data Preprocessing}

Since the combined resolution of all the heightmaps was excessively large for the scale of the envisioned map, they were downsized to a more manageable file size and stitched together and through QGIS (2017).

New Zealand coastlines were extracted from the vector world map acquired from Natural Earth (NACIS, 2017) projected to Pseudo Mercator and exported to Al format.

\section{Modelling}

In Houdini (SideFX, 2017) metadata can be stored as attributes on 3D elements such as models, polygons, vertices or points. Points being simple locations in space which can be used to position or build geometrical objects. When importing a CSV table, a point is created for every line, and selected fields can be passed as attributes. In this way, propitious variables such as date, magnitude, depth, latitude and longitude can be stored.

As Houdini doesn't natively support map projections, code based on the WGS 84/Pseudo-Mercator formula was written to accurately transform latitude and longitude to XZ coordinates, making it possible to accurately work with decimal degrees throughout the workflow.

Due to the large area of open sea surrounding New Zealand, extruding elevation from a rectangular plane would result in a lot of unnecessary geometry and wouldn't display coastlines. Instead, the meta-operator for extrusion was modified to process any vector file input into a polygonal surface, resample it depending on a resolution parameter and proceed with the heightmap extrusion. The 
adjustable resolution makes it possible to work with an optimised version of the mesh during production.

By this stage, a major part of the visualisation workflow can be packaged into a single meta-operator. This asset takes the following inputs:

- A heightmap of the terrain and a vector file of the region to be extruded, cropped to the same extent.

- A CSV table with at least latitude and longitude coordinates.

- Variables for the boundary coordinates and the zoom scale of the map.

- Variable for the resolution.

The operator outputs a 3D mesh and an array of points with meta-data from the CSV accurately located on its surface.

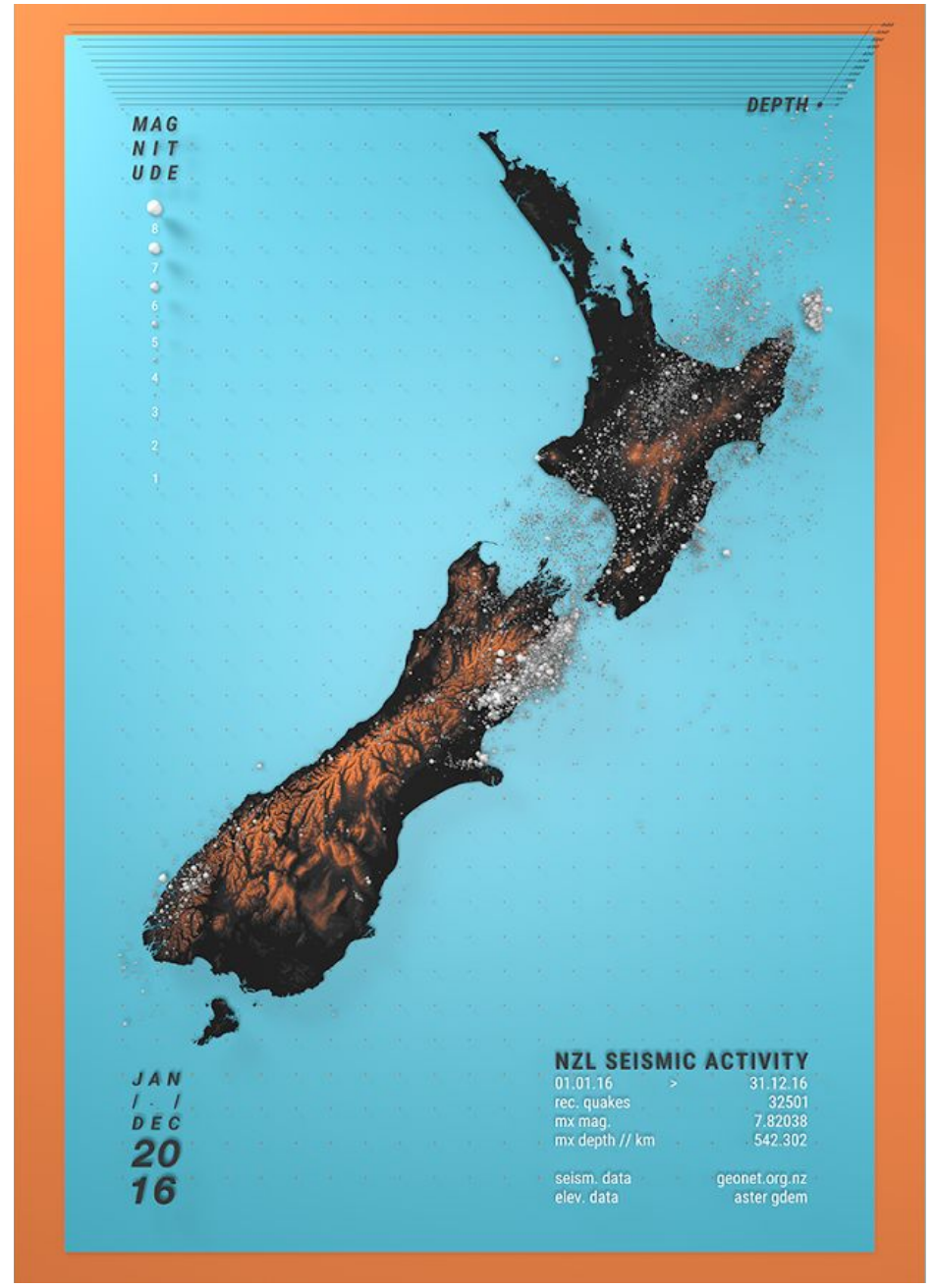

Figure 5. NZL Seismic Activity 1. 
With the essential modelling complete, the iterative development and refinement of content specific atypical geo visualisations begun. It was safely assumed that earthquake depth would be most intuitively displayed by displacing points along the $Y$ axis, and while some code was written to place the objects at their actual depth based on the scale and projection of the map, the small scale and density of points made it difficult to discern patterns in the data. Instead, the depth was exaggerated, and a graduated scale was added. The terrain elevation was similarly accentuated to be more legible at such a scale, and a height multiplier variable was added to the corresponding meta-operator.

As we instinctively compare the volume of objects, visualising the magnitude of earthquakes through accordingly scaled spheres was an obvious decision. Nonetheless, alternatives were experimented with.

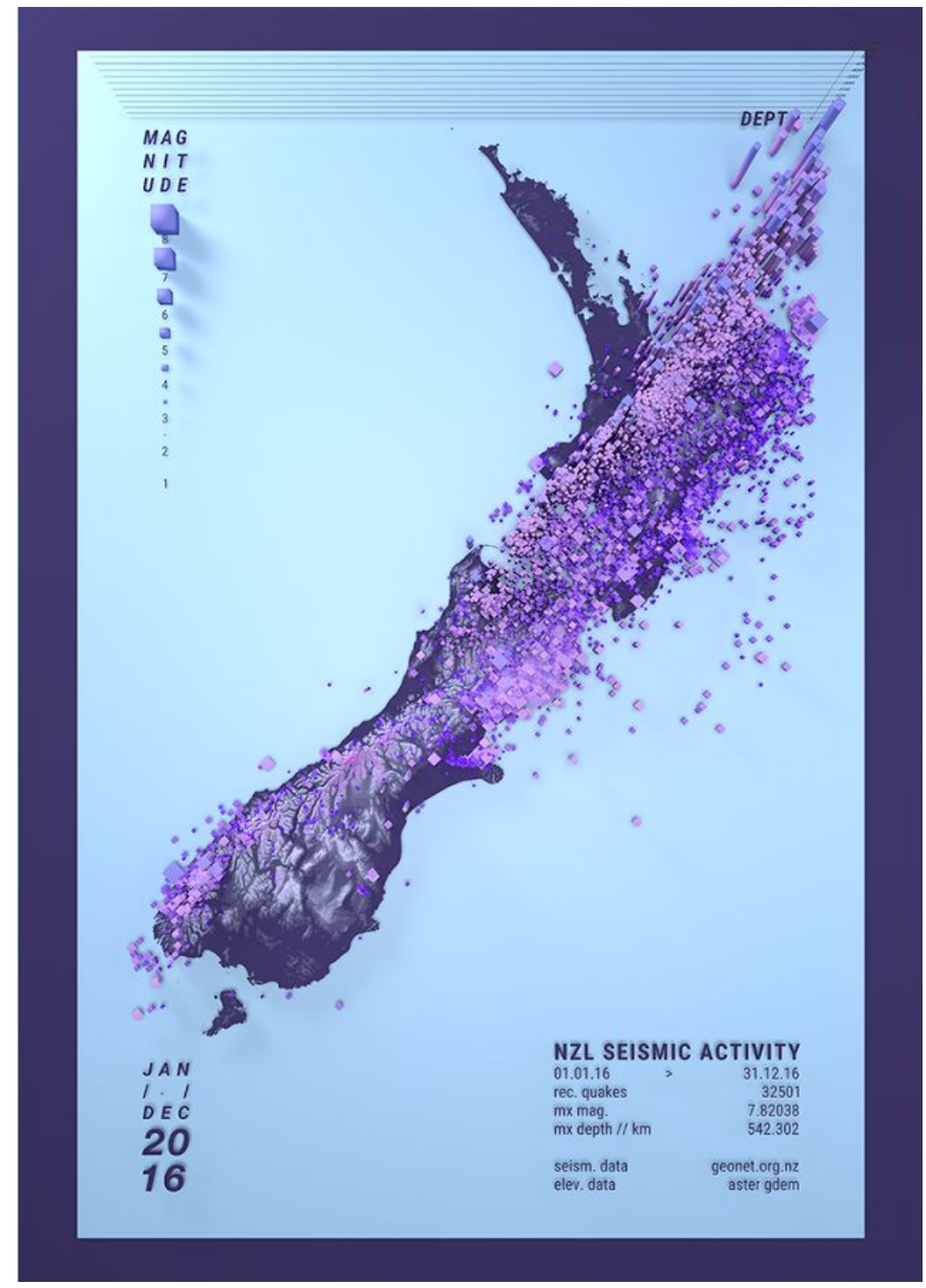

Figure 6. NZL Seismic Activity 2. 
While depth should realistically be visualised below the terrain mesh, finding viewing angles where the data-points weren't obscured was difficult. This could be remedied by making the terrain transparent or visualised as a floating dot matrix, but in the process sacrificing a major graphical element of the composition. Displaying depth above the mesh was less intuitive but more legible and visually compelling.

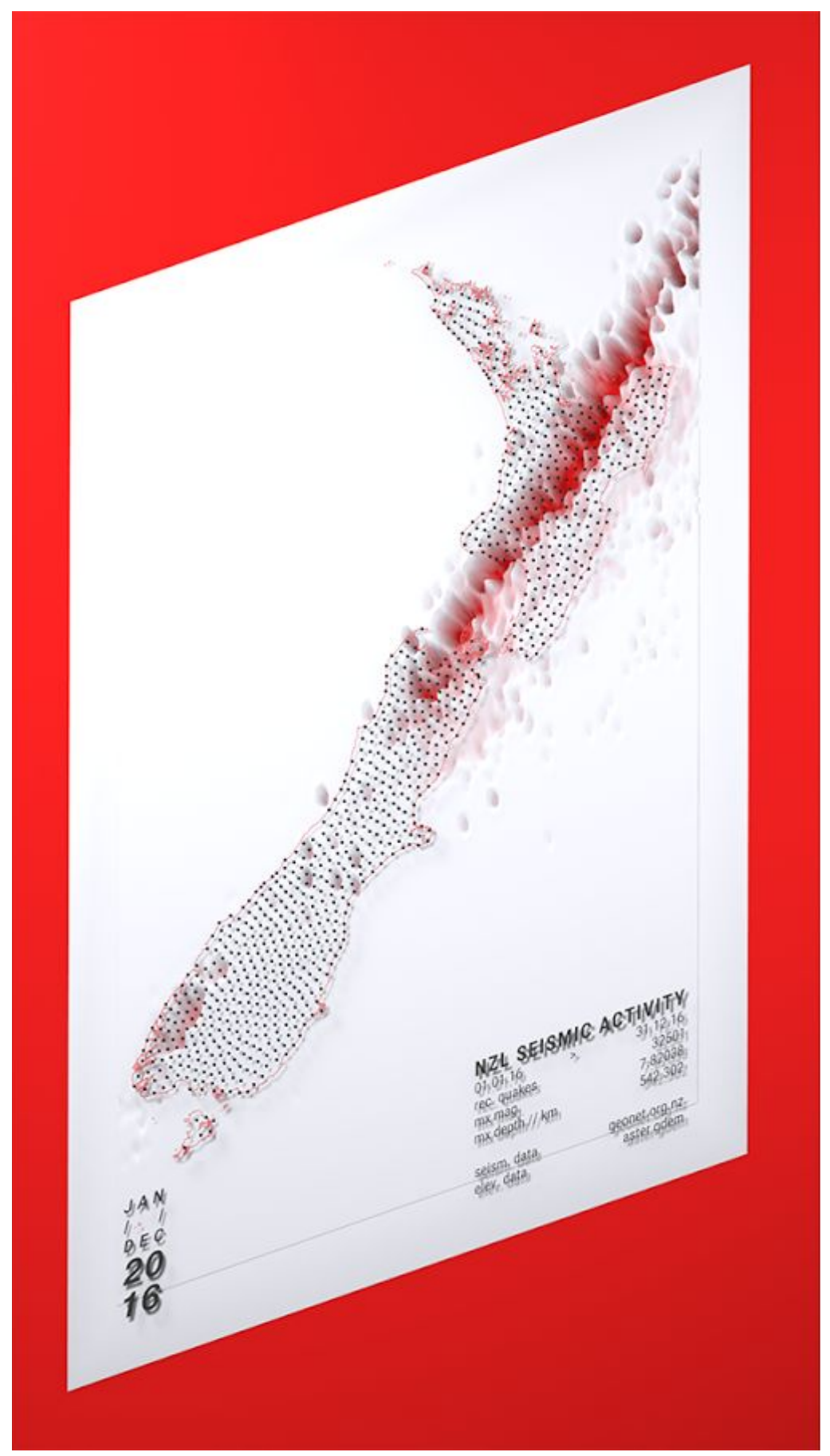

Figure 7. NZL Seismic Activity 3. 
Rather than displaying every data-point as individual objects, a method of using them to procedurally create 3D artefacts was investigated. A promising attempt consisted of deforming a subdivided plane based on the average depth of earthquakes at each vertex location, effectively creating an earthquake heightmap. This visualisation was quite limited however as it did not account for the density or magnitude of earthquakes and would have to either be displayed below the mesh or hide underlying terrain.

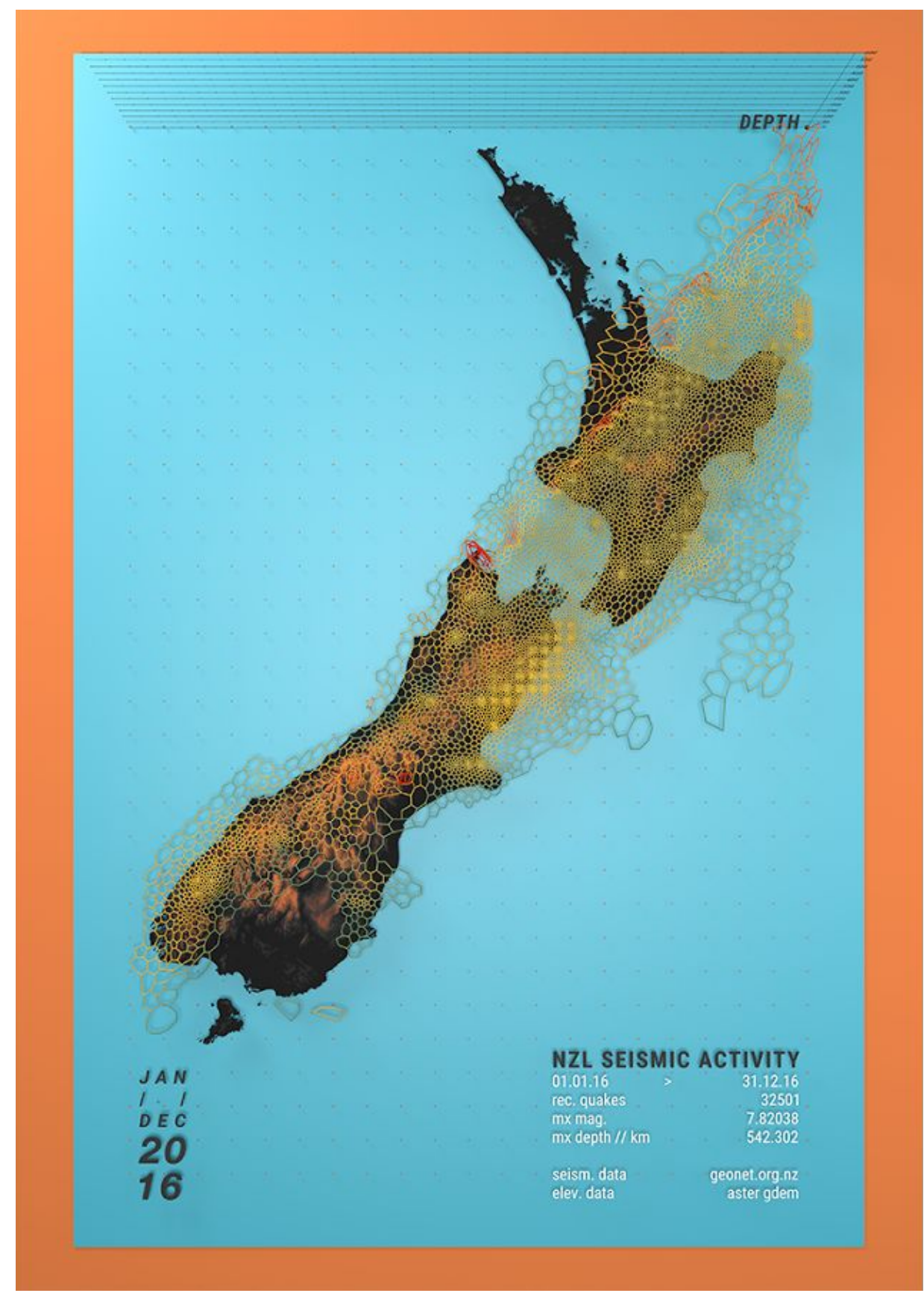

Figure 8. NZL Seismic Activity 4. 
This visibility issue can be remedied by remeshing the geometry into a Voronoi wireframe sitting atop the terrain, the size of the cells corresponding to the density of earthquakes. Average magnitude can then be displayed as a colour heatmap over the mesh resulting in an aesthetically engaging visualisation. By iteratively varying variables such as the mesh resolution, colour ramp and falloff distance, patterns are highlighted in intriguing and unconventional ways.

\section{Stylisation}

As covered in the literature review, good infographics should favour legibility, brevity, and hierarchically organise text information. To implement these theories a graphical style had to be devised to inform the design of text labels, colour schemes and composition.

Swiss Style, also known as International Typographic Style, strongly informed this design approach, as its theoretically focus on clarity, simplicity, minimalism and objectivity has historically been extremely influential to the development of infographics and graphic design in general (Hollis, 2006).

Concretely, sans-serif typefaces were preferred for their restrained yet aesthetically rich forms, and were contrasted by varying size, case and weight. Strong and bold colours were deliberately utilised to differentiate backgrounds and elements, making for appealing, eye-catching compositions.

\section{Visualisation}

In order to smoothly illuminate the geometry many light setups were experimented with. A relatively straightforward three-point light setup was found to give the best results, with one stronger high angle light positioned so as to cast soft and subtle shadows, highlighting the three-dimensionality of the maps even when viewed from above. 


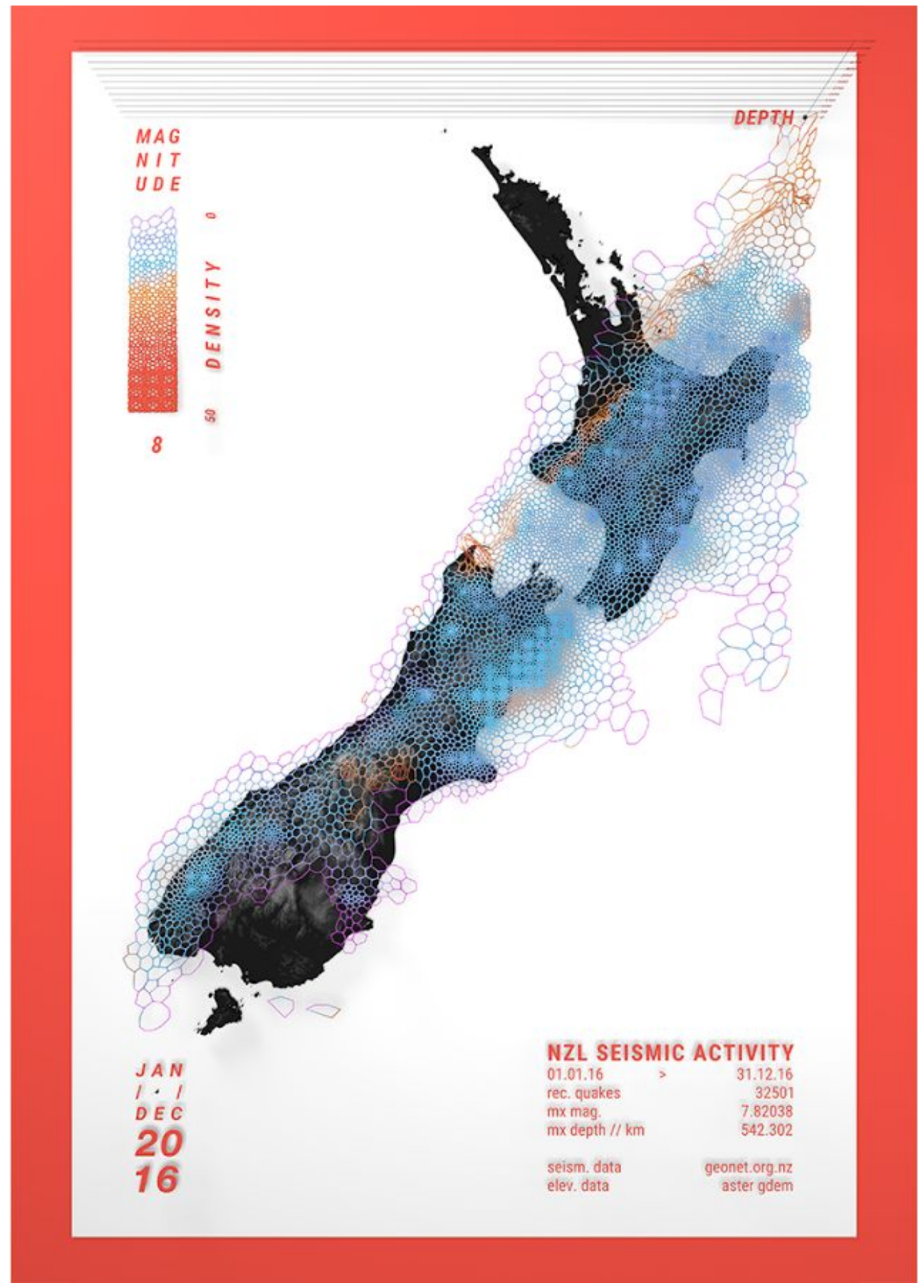

Figure 9. NZL Seismic Activity 5.

As various viewing angles were experimented with, top down angles were found to give the most accurate visualisation of the geolocations, as the perspective distortion was negligible and most objects were mostly unobstructed. The trade-off was inferior comprehension of the height component of the display. 


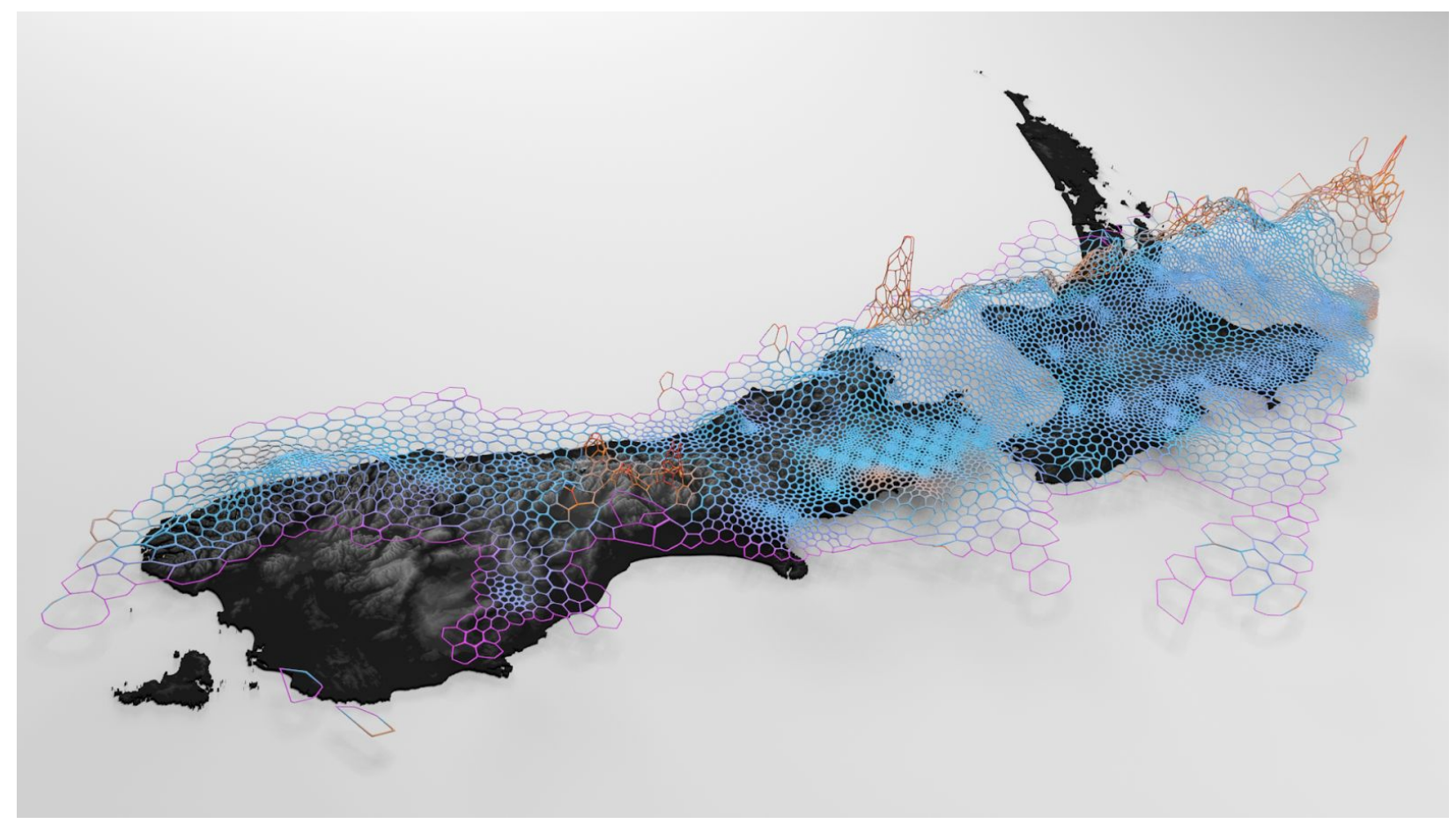

Figure 10. NZL Seismic Activity 6.

Medium to high angle perspective shots were most dramatic and immersive, giving an intuitive view of elevation, but suffered the most from perspective deformation and obstruction issues. 


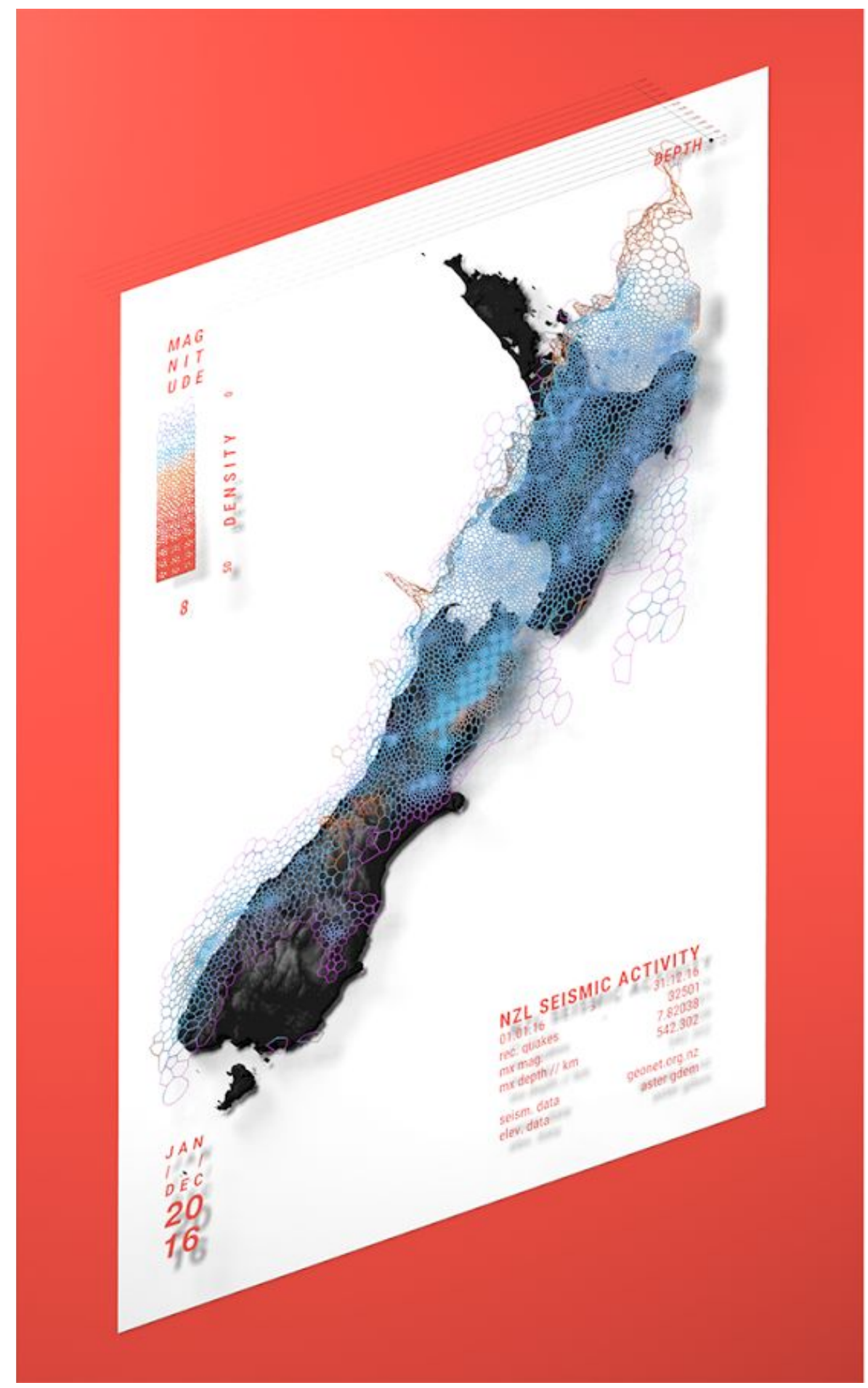

Figure 11. NZL Seismic Activity 7.

Isometric style orthographic views offer a good compromise between the two, doing away with perspective distortion and reducing obstruction while still giving a good view of the elevation. This comes at the cost of a making the panorama less natural and immersive. 


\section{United States and Mexico Meteorite Landings Geo-Visualisation}

\section{Data selection}

Keeping with the theme of natural phenomenon a CSV table of the Meteorite Landings Bulletin (The Meteoritical Society, 2015) was acquired from the NASA Open Data Portal (Nasa, 2017). This dataset comprises the landing coordinates, year, mass and a name identifier for every recorded meteorite fall.

As a preliminary visualisation showed good data density in mainland America and Mexico, elevation data for the region had to be acquired. Due to the massive extent of the region, selecting and downloading over 1.6 thousand fragments through the EarthExplorer (USGS, 2017) web-app and batch downloader would be terribly tedious. Instead, the data can be easily selected and ordered from the NASA's EarthData website (NASA, 2017). Once the request has been processed, a weblink to an archive of the desired data is received through email. The height maps can then be bulk downloaded with a simple web-scraping python script. This method should be favoured when working at a continental scale as the download speed is vastly superior to the EarthExplorer (USGS, 2017) repository.

Wanting to experiment with raster textures, a hypsometric tint world map was also acquired from the Natural Earth (NACIS, 2017) site.

\section{Data Preprocessing}

The bulk of the data, over 60GB, proved too much for the QGIS (2017) graphic interface to handle; instead, a procedural GDAL script was written and executed in the OSGeo4 PowerShell packaged with the software. Given a maximum display size variable and folder location, the script resizes fragments individually before stitching and projecting them as a reasonably sized Pseudo-Mercator heightmap. 


\section{Modelling}

Visualising the meteorites as spheres of varying scale based on recorded mass seemed thematically appropriate, however, due to the drastic difference in mass, ranging from a few grams to many tonnes, colossal meteors would obstruct numerous smaller ones and the underlying terrain.

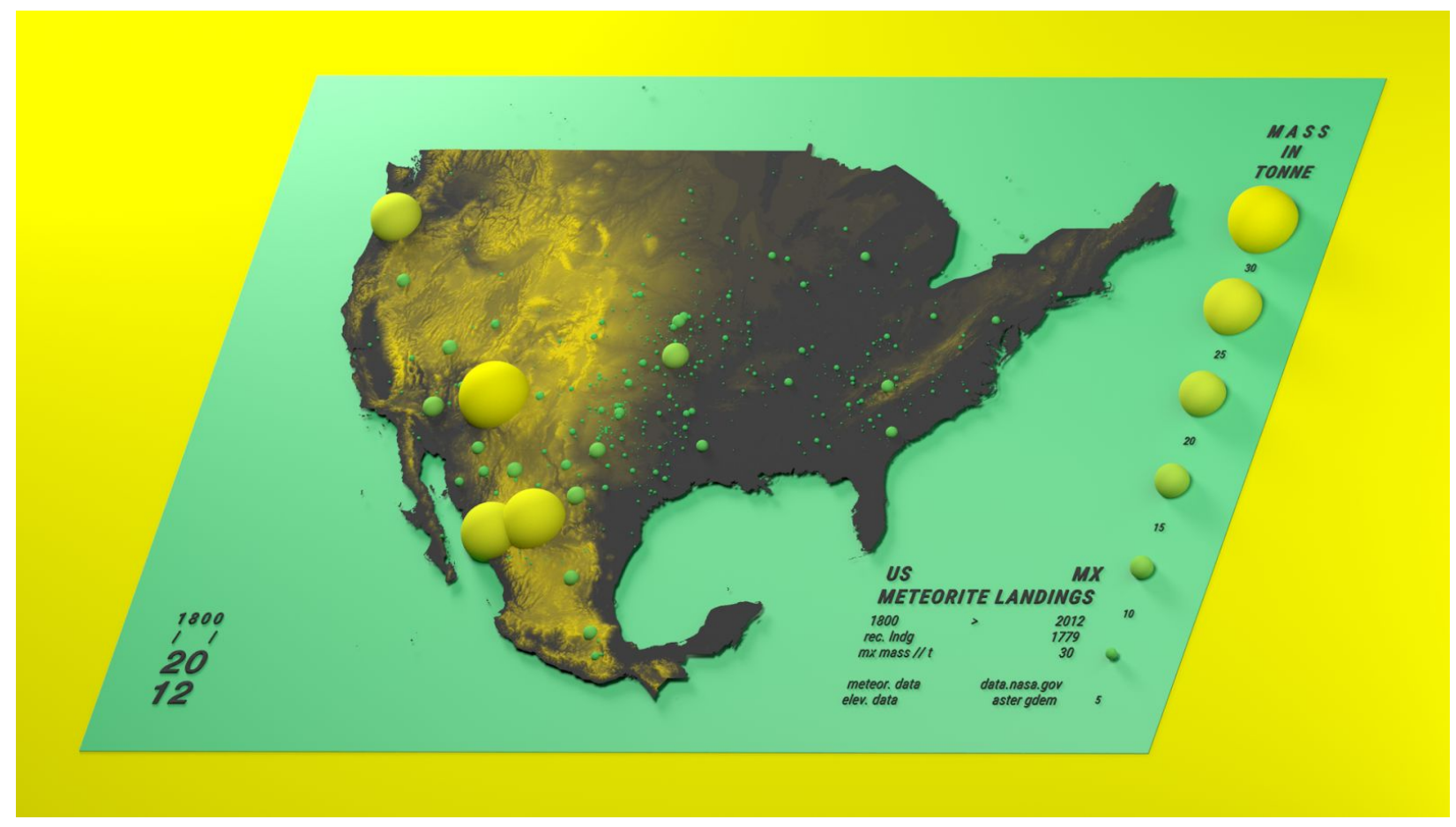

Figure 12. US - MX Meteorite Landings 1.

By experimenting with boolean operation, landing sites can be intuitively visualised as craters. This type of operation performed on medium and high res meshes proved to be very computationally intensive, in turn significantly limiting iteration speed. Unfortunately, more massive meteorites erased a lot of smaller data-points. 


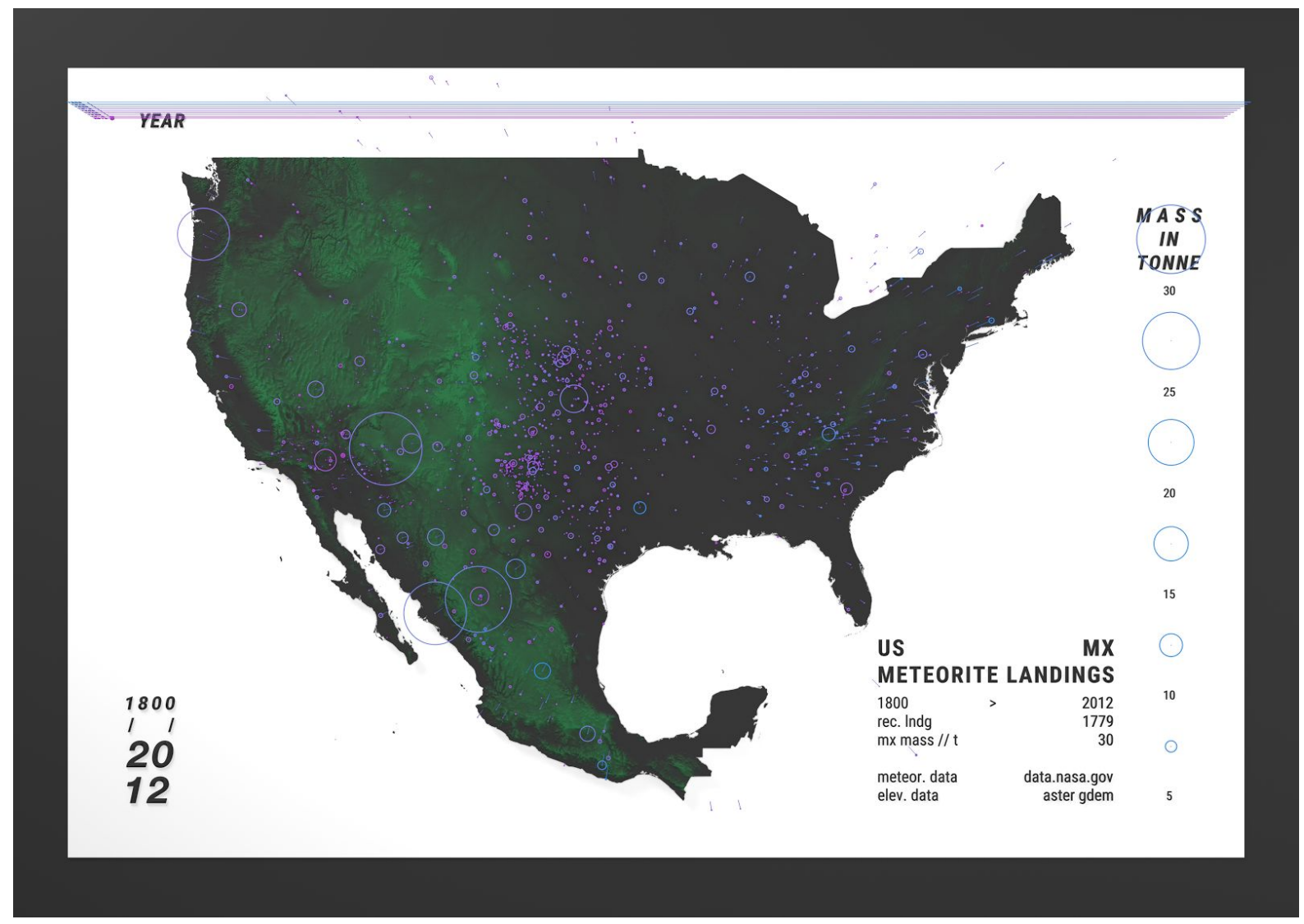

Figure 13. US - MX Meteorite Landings 2.

Using rings instead offered excellent legibility at much lesser computational cost. As humans visually compare the size of two-dimensional circles based on their surface area rather than their diameter (Krum, 2013), the code was correspondingly adjusted so as to provide accurate and intuitive comparative visualisation of mass. With the visibility afforded by hollow rings, the $Y$ axis was utilised as a visualisation of time, with older landings further away from the surface of the mesh. This was reinforced with a colour component for easier readability. 


\section{Stylisation}

In order to showcase a more complete and natural visualisation of the topography, a climate adjusted hypsometric map was projected on the surface. Unlike monochromatic heightmaps, the hypsometric map adjusts the tint based on both elevation and type of terrain, in this way arid areas are colored with earth tones, humid lowlands in shades of green and snow-capped mountains in white.

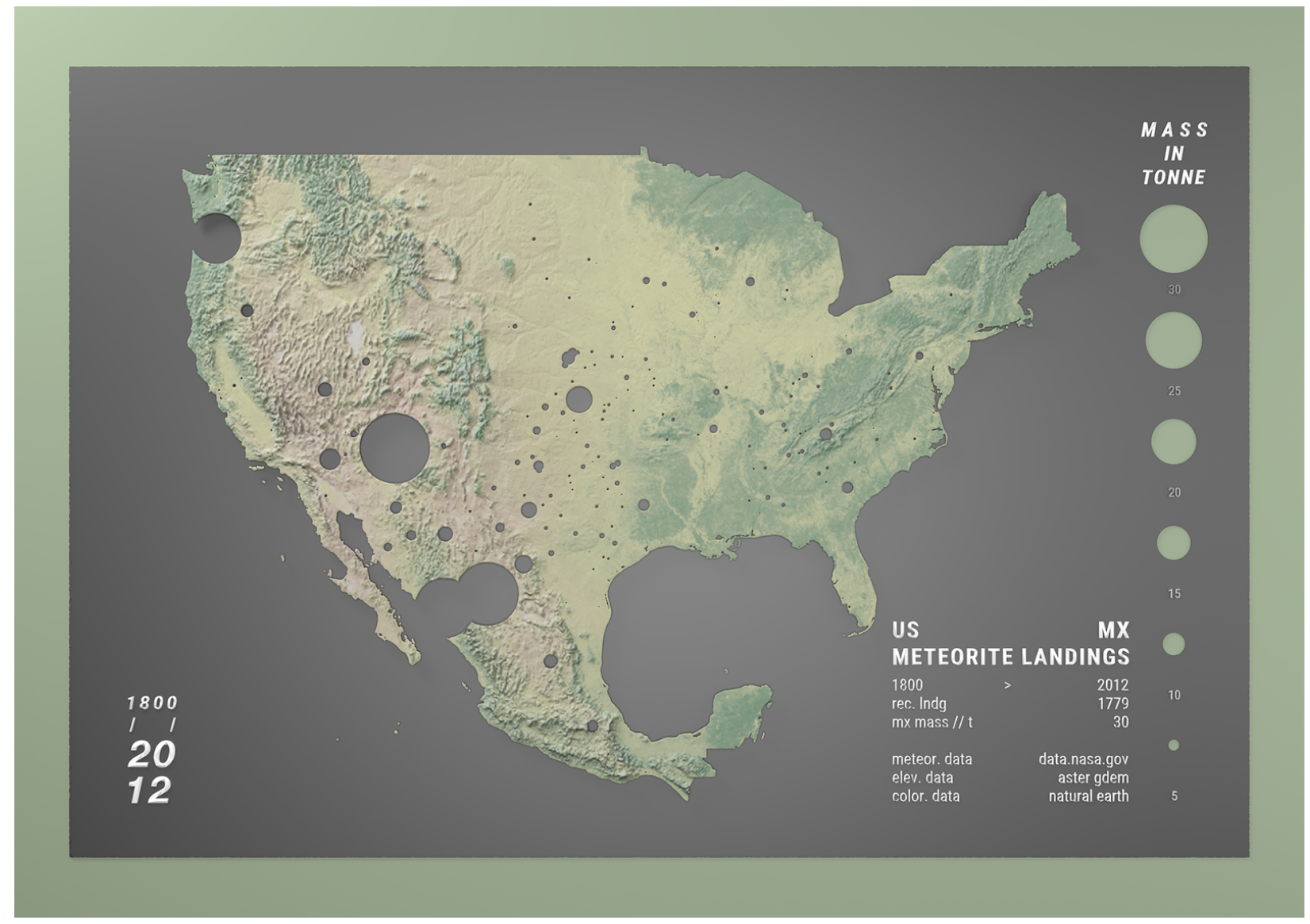

Figure 14. US - MX Meteorite Landings 3. 


\section{Twitter Data Mining Workflow}

\section{Data selection}

Just as natural earth data makes for interesting and beautiful visualisations of the workings of our planet and its place in the universe, user-generated social media content has the potential to highlight patterns in populations and their use of social media. The Twitter social platform is unique in that it's strict word-limit and content sharing structure favours the circulation of passing thoughts, quips, commentary and spontaneous communications (Russell, 2013). This short format makes the platform extremely active and users can tweet from geotagging capable phones or tablets as they go about their day. This wealth of geolocated information combined with an easily parsable metadata format and extensive API support made it admirably predisposed to geo-data-mining and visualisation.

The Tweepy library for Python enables researchers to relatively easily and efficiently scrape twitter through their proprietary API. However, a number of factors must be taken into consideration when utilising the API to mine data. Most importantly the API search is limited in time, tweets older than nine days won't be returned from a search by keyword or geo-coordinates, and the number of tweets that can be accessed per seconds is prone to significant throttling. Only a small percentage of users share their location, and fewer still geotag their content. From the tweets mined by keyword search in this study, only $27 \%$ had location data such as town or suburb, and a meagre $0.2 \%$ were tagged with latitude and longitude coordinates.

These limitations mean that to gain suitably large data sets, the scraping script must run for extended periods of times. As the API does not allow to search by location and keyword simultaneously, the data must first be saved to a JSON file before location data can be extracted.

While it is tempting to leave the listener running for multiple days to acquire massive data sets, when doing so, frequent and unpredictable data corruption issues were encountered, and the huge size of the files produced was slow and very resource intensive to work with.

\section{Data Preprocessing and Enrichment}

As the complete twitter meta-data is saved in a JSON file, relevant information can be extracted. To do this, a python script was written to iterate over all tweets, check for and retrieve location information if available. Given the rarity of tweets' specific coordinates, the script also checks for the user's profile location if provided. All other tweets are scrapped.

While the data is now trimmed to only relevant information, it still in an unsuitable format, with most tweets lacking latitude and longitude coordinates, as most users only provide addresses or city names. Thanks to the Google Maps (Google, 2005) and OpenStreetMap (OpenStreetMap Foundation, 2004) geocoding APIs, a request can be sent with an address or location name and return a comprehensive JSON response including lat-long coordinates. This geocoding can be done for any data sets including location names, although artefacts may happen due to multiple places sharing the same names. 
Another python script was developed to encode location data into geocoding request URLS and parse the JSON response for coordinates. The recovered data is then formatted into a CSV file using a language module for python. This data enrichment process gives a good approximation of tweets' geographical provenance.

\section{United States Patriotic Tweets Adjusted by State Population}

With the ability to mine twitter for geolocation, it was possible to combine and juxtapose user generated information with geodata and adjust it based on population information acquired from census data. Using the process outlined above, a Twitter scrape query of hashtags associated with US patriotism was used to mine and enrich a set of tweet locations.

Vector data was once again acquired from the Natural Earth (NACIS, 2017) website, this time a more detailed file comprising individual states. While this data-sets includes metadata such as state names, a downside of the import process to Houdini is the loss of such data. This can however easily be remedied by enriching a list of states addresses using the same geocoding process used to process tweets, and projecting the data back onto the geometry based on proximity. This same process can be used to enrich any other data pertaining to a place or region, such as the population estimates acquired from the United States Census Bureau (2016). 


\section{Modelling}

Once all data had been enriched and imported, a reusable script operator was written to project any data from geolocated points to a regional mesh. In this way, the number of tweets could be procedurally counted by state boundary and saved into the metadata of the polygons. The total number of patriotic tweets can then be visualised by extruding states proportionally.

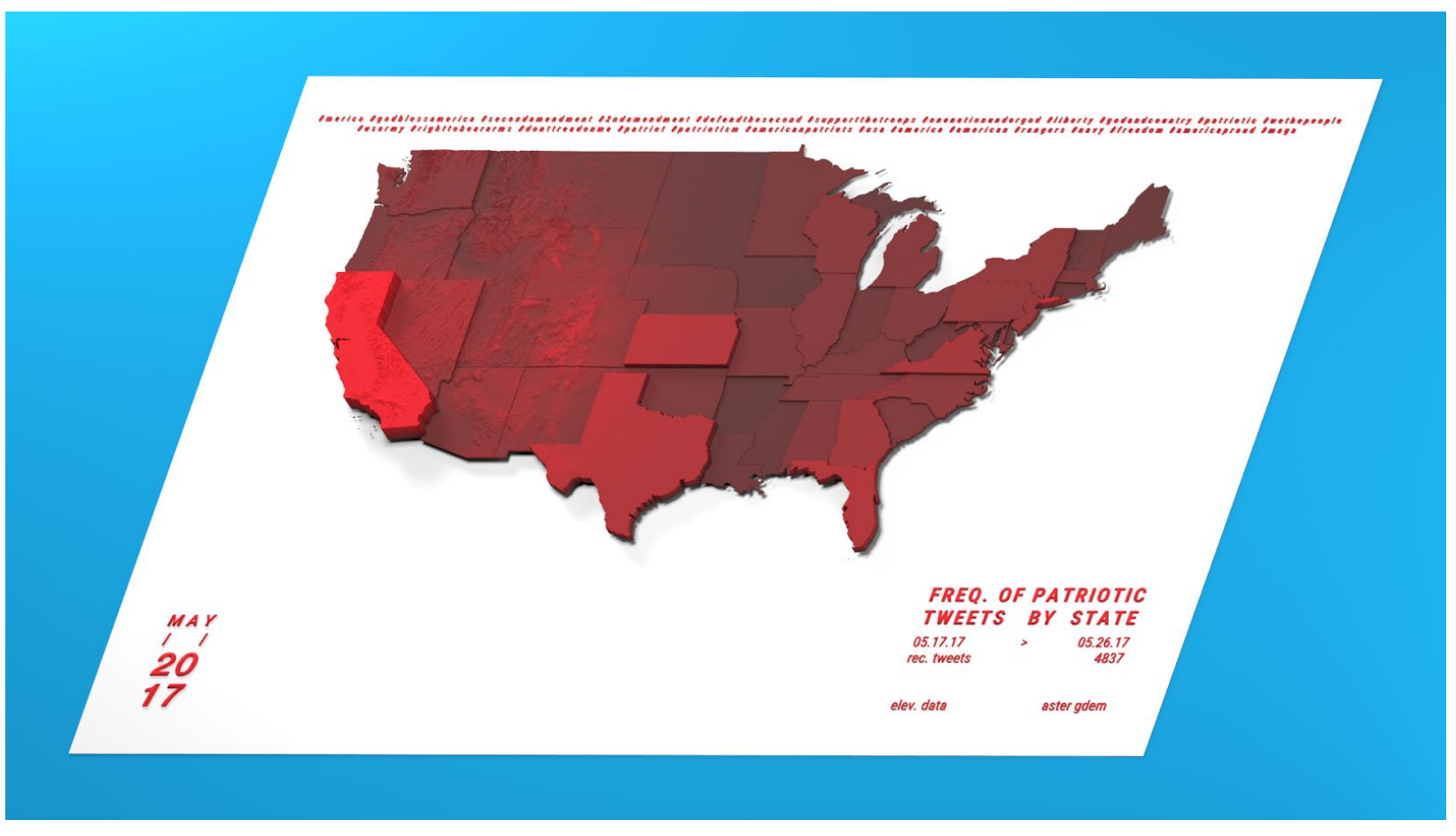

Figure 15. Frequency of Patriotic Tweets by State. 
However since this doesn't account for state population, the visualisation could be communicating a skewed perspective of reality as it doesn't give a faithful presentation of per capita twitter patriotism. It is easily remedied however by projecting the population data from the Annual Estimates of the Resident Population for the United States (United States Census Bureau, 2016) and normalising the value. This drastically alters the visualisation, offering a more meaningful and less deceptive view of the concentration of American patriots by state.

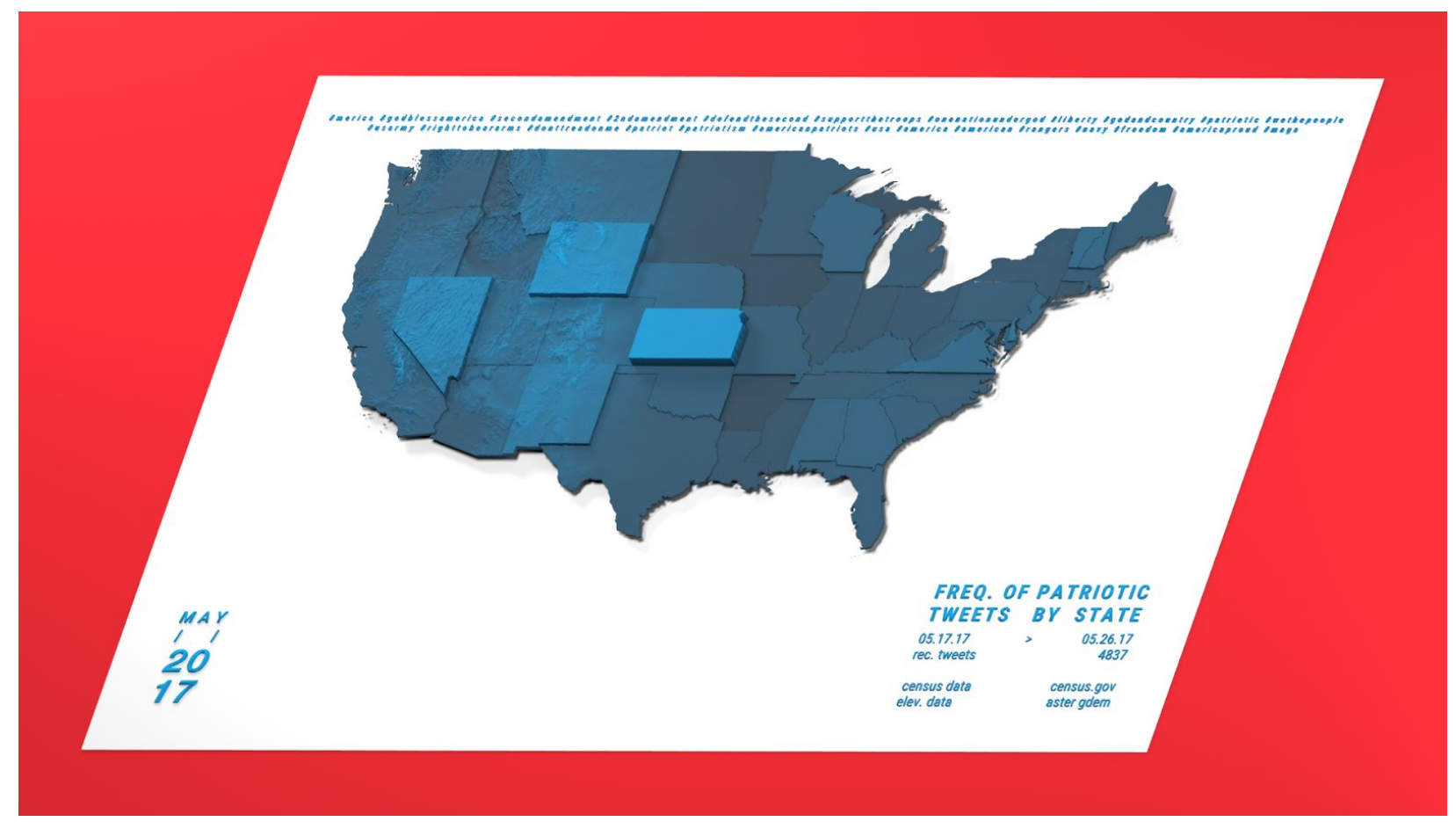

Figure 16. Normalised Frequency of Patriotic Tweets by State. 


\section{Conclusion}

Three-dimensional geo-visualisation is still a nascent field deeply rooted in GIS and its predominantly scientific origin, however, it's innate immersivity and potential to astonish audiences also make it an alluring context for aesthetically conscious data-visualisations and infographic designs aimed at lay people. With the prospect of increased multidisciplinary appeal, it is critical for workflows and design precedents to be established so as to facilitate future contribution to this growing knowledge pool. It can be anticipated that the tacit knowledge and design conventions contemplated through experiments such as the ones documented herein might eventually propagate to other more scientific geo-visualisations contexts. Such an outcome could eventually raise the visual quality and legibility of 3D infocartographics across disciplines, and popularise 3D maps in a process analogous to the spread of web infographics.

While automation is quintessential to the processing of large data-sets, in order to produce unique and deliberate cartographic artefacts, extensive control must be maintained throughout the data transformation process. The flexible and procedural visual coding environment of Houdini (SideFX, 2017) was found to offer excellent support for user-defined automation and aesthetic control. The workflow built around this procedural paradigm provides an actionable pipeline for the acquisition, enrichment, processing, modelling, stylisation and visualisation of data from various online repositories and social media platforms.

The aesthetic and compositional features of the produced artefacts can be examined and critiqued to better inform future geo-visualisation projects. Indeed there remain many opportunities for further development and applications of the tools built in this workflow. While the format of the design output is befitting of digital and print media, the appreciation of the elements is limited by the pre-rendered viewing angles. With small adjustments and optimisation to the geometry, the production pipeline could be expanded for physical 3D printing or augmented reality deployment. 


\section{References}

\section{Software}

Side Effects Software. (2017) Houdini (Version 16.0.557) [3D computer graphics software]. Toronto, Canada: SideFX.

Autodesk. (2017) Maya (Version 2017) [3D computer graphics software]. San Rafael, California: Autodesk.

Epic Games. (2017) Unreal Engine (Version 4.16) [3D Game Engine]. Cary, North Carolina: Epic Games.

Solid Angle. (2017) Arnold (Version 2.0.1) [Rendering system]. Madrid, Spain: Solid Angle.

QGIS Development Team. (2017). QGIS (Version 2.18.10 ) [Geographic information system software]. Retrieved from http://qgis.org

Google. (2005). Google Maps [Mapping site]. Retrieved from https://maps.google.com

Twitter Inc. (2006). Twitter [Social network site]. Retrieved from https://twitter.com

OpenStreetMap Foundation. (2004). OpenStreetMap [Collaborative mapping site]. Retrieved from https://www.openstreetmap.org

\section{Database}

North American Cartographic Information Society. (2017) Natural Earth [Database].Retrieved from http://naturalearthdata.com/

NASA LP DAAC, METI. (2011). ASTER Global Digital Elevation Model (2) [Database].Retrieved from https://lpdaac.usgs.gov

United States Geological Survey. (2017). USGS EarthExplorer [Online data discovery tool]. Retrieved from https://earthexplorer.usgs.gov

GNS Science, Earthquake Commission. (2017). GeoNet Earthquake Catalogue[Database]. Retrieved from https://geonet.org.nz

NASA. (2017). NASA's Open Data Portal[Database]. Retrieved from https://data.nasa.gov

NASA. (2017). Earth Observation Data[Database]. Retrieved from https://earthdata.nasa.gov

The Meteoritical Society. (2015). Meteorite Landings Bulletin [Data Set]. Retrieved from https://data.nasa.gov/Space-Science/Meteorite-Landings/gh4g-9sfh 
United States Census Bureau. (2016) Annual Estimates of the Resident Population for the United States, Regions, States, and Puerto Rico: April 1, 2010 to July 1, 2016 [Data Set]. Retrieved from https:/census.gov/data/tables/2016/demo/popest/state-total.htm

\section{Literature}

Bandrova, T. (2005). Innovative technology for the creation of 3D maps. Data Science Journal, 4, 53-58.

Hilbert, M. (2012). How much information is there in the "information society"?. Significance, 9(4), 8-12.

Smith, A. (2013). Emerging Trends in Data Visualisation: Implications for Producers of Official Statistics. In X. He (Ed.) Proceedings 59th ISI World Statistics Congress. The Hague, The Netherlands: International Statistical Institute.

Perkins, C. (2002). Cartography: progress in tactile mapping. Progress in Human Geography, 26(4), 521-530.

Ormeling, F. (2014). Cartography as Intentional Distortion. In Thematic Cartography for the Society (pp. 341-357). Springer International Publishing.

Goodchild, M. (2012). Cartography: GIS and cartography (relationship between). Santa Barbara, CA.

Cartwright, W. (2009). Art and Cartographic Communication. Cartography and Art, 23-12.

Cartwright, W., Gartner, G., \& Lehn, A. (Eds.). (2009). Cartography and art. Springer Science \& Business Media.

Fairbairn, D. (2009). Rejecting illusionism: transforming space into maps and into art. Cartography and Art, 23-34.

Hájek, P., Jedlička, K., Kepka, M., Fiala, R., Vichrová, M., Janečka, K., \& Čada, V. (2015). 3D cartography as a platform for remindering important historical events: the example of the Terezín memorial. In Modern Trends in Cartography (pp. 425-437). Springer International Publishing.

Howe, S. G., \& Ruggles, S. P. (1837). Map of Florida. Atlas of the united states, Printed for the use of the blind. Boston, MA: New England Institution for the Education of the Blind.

Pegg, D. (2013). Design issues with 3D maps and the need for 3D cartographic design principles.

Häberling, C., Bär, H., \& Hurni, L. (2008). Proposed cartographic design principles for 3D maps: a contribution to an extended cartographic theory. Cartographica: The International Journal for Geographic Information and Geovisualization, 43(3), 175-188.

Schobesberger, D., \& Patterson, T. (2007). Evaluating the effectiveness of $2 d$ vs. $3 d$ trailhead maps. Mountain Mapping and Visualisation, 201. 
Semmo, A., Trapp, M., Jobst, M., \& Döllner, J. (2015). Cartography-Oriented Design of 3D Geospatial Information Visualization-Overview and Techniques. The Cartographic Journal, 52(2), 95-106.

Krum, R. (2013). Cool infographics: Effective communication with data visualization and design. John Wiley \& Sons.

Weinreich, H., Obendorf, H., Herder, E., \& Mayer, M. (2008). Not quite the average: An empirical study of Web use. ACM Transactions on the Web (TWEB), 2(1), 1-31.

Bellato, N. (2013). Infographics: A visual link to learning. ELearn, 2013(12), 1.

Nelson, D. L., Reed, V. S., \& Walling, J. R. (1976). Pictorial superiority effect. Journal of Experimental Psychology: Human Learning and Memory, 2(5), 523.

Groenendyk, M. (2013). Emerging Data Visualization Technologies for Map and Geography Libraries: 3-D Printing, Holographic Imaging, 3-D City Models, and 3-D Model-based Animations. Journal of Map \& Geography Libraries, 9(3), 220-238.

Mao, B. (2011). Visualisation and generalisation of 3D City Models (Doctoral dissertation, KTH Royal Institute of Technology).

Ramachandran, P., \& Varoquaux, G. (2011). Mayavi: 3D Visualization of Scientific Data. Computing in Science \& Engineering, 13(2), 40-51.

Helbig, C., Bauer, H., Rink, K., Wulfmeyer, V., Frank, M., \& Kolditz, O. (2014). Concept and workflow for $3 \mathrm{D}$ visualization of atmospheric data in a virtual reality environment for analytical approaches. Environmental Earth Sciences, 72(10), 3767-3780.

Miller, H. J., \& Han, J. (Eds.). (2009). Geographic data mining and knowledge discovery. CRC Press.

Russell, M. A. (2013). Mining the Social Web: Data Mining Facebook, Twitter, LinkedIn, Google+, GitHub, and More. " O'Reilly Media, Inc.".

Zafarani, R., Abbasi, M. A., \& Liu, H. (2014). Social media mining: an introduction. Cambridge University Press.

ComScore. (2017). 2017 U.S. Cross-Platform Future in Focus. Retrieved from https://www.comscore.com/Insights/Presentations-and-Whitepapers/2017/2017-US-Cross-PlatformFuture-in-Focus

Cooley, M. (2000). Human-centered design. Information design, 59-81.

Gedenryd, H. (1998). How designers work-making sense of authentic cognitive activities (Vol. 75). Cognitive Science.

Biggs, M. A., \& Büchler, D. (2007). Rigor and practice-based research. Design issues, 23(3), 62-69. 
Zimmerman, J., Forlizzi, J., \& Evenson, S. (2007, April). Research through design as a method for interaction design research in $\mathrm{HCl}$. In Proceedings of the SIGCHI conference on Human factors in computing systems (pp. 493-502). ACM.

McNiff, J. (2013). Action research: Principles and practice. Routledge.

Pedgley, O. (2007). Capturing and analysing own design activity. Design Studies, 28(5), 463-483.

Corbin, J., \& Strauss, A. (1990). Grounded theory research: Procedures, canons and evaluative criteria. Zeitschrift für Soziologie, 19(6), 418-427.

Findeli, A. (2004, May). La recherche-projet: une méthode pour la recherche en design. In Texte de la communication présentée au premier Symposium de recherche sur le design à HGK de Bâle sous les auspices du Swiss Design Network, Bâle.

Durrant, A., Vines, J., Wallace, J., \& Yee, J. (2015). Developing a Dialogical Platform for Disseminating Research through Design. Constructivist Foundations, 11(1), 8-21.

Creswell, J. W. (2013). Research design: Qualitative, quantitative, and mixed methods approaches. Sage Publications.

Norman, D., \& Verganti, R. (2014). Incremental and radical innovation: Design research vs. technology and meaning change. Design Issues, 30(1), 78-96.

Koskinen, I., Zimmerman, J., Binder, T., Redstrom, J., \& Wensveen, S. (2011). Design research through practice: From the lab, field, and showroom. Elsevier.

Norman, D. A. (2005). Human-centered design considered harmful. interactions, 12(4), 14-19.

Godin, D., \& Zahedi, M. (2014). Aspects of research through design: a literature review. Proceedings of DRS, 281.

Hollis, R. (2006). Swiss graphic design: the origins and growth of an international style, 1920-1965. Laurence King Publishing.

Miller, H. J., \& Han, J. (Eds.). (2009). Geographic data mining and knowledge discovery. CRC Press. 